\title{
Immersion and Invariance Adaptive Control for Spacecraft Pose Tracking via Dual Quaternions
}

\author{
Xiaoping Shi $\mathbb{D},{ }^{1}$ Xuan Peng $\mathbb{D},{ }^{1}$ and Yupeng Gong $\mathbb{C}^{2}$ \\ ${ }^{1}$ Control and Simulation Center, Harbin Institute of Technology, Harbin 150080, China \\ ${ }^{2}$ Research Center of Satellite Technology, Harbin Institute of Technology, Harbin 150080, China \\ Correspondence should be addressed to Xuan Peng; px_hit@126.com
}

Received 2 December 2020; Revised 20 January 2021; Accepted 25 February 2021; Published 16 April 2021

Academic Editor: Thach Ngoc Dinh

Copyright (c) 2021 Xiaoping Shi et al. This is an open access article distributed under the Creative Commons Attribution License, which permits unrestricted use, distribution, and reproduction in any medium, provided the original work is properly cited.

This paper addresses the simultaneous attitude and position tracking of a target spacecraft in the presence of general unknown bounded disturbances in the framework of dual quaternions, which provides a concise and integrated description of the coupled rotational and translational motions. By virtue of the newly introduced dual direction cosine matrix, the dimension of the dual quaternion-based relative motion dynamics written in vector/matrix form can be lowered to six. Treating the disturbances as unknown parameters, a modular adaptive pose tracking control scheme composed of two separately designed parts is then derived. One part is the adaptive disturbance estimator designed based on the immersion and invariance theory. Driven by the disturbance estimation errors, it can realize exponential convergence of the estimations and has the nice "parameter lock" property, which can hardly be expected in the conventional certainty equivalent adaptive controllers. The other part is a proportional-derivative-like pose tracking controller where the estimated disturbances are directly used. The closed-loop stability of the relative motion system under different kinds of disturbances is proven by Lyapunov stability analysis. Simulations and comparisons with two previous dual quaternion-based controllers demonstrate the novel features and performance improvements of the proposed control scheme.

\section{Introduction}

Simultaneously tracking the desired attitude and position (pose) with respect to (w.r.t) a target spacecraft with high precision is a crucial and indispensable technology for a broad range of space proximity missions, such as on-orbit service, rendezvous and docking, and so on [1-4]. This problem is intractable since it necessitates the six-degree-of-freedom (six-DOF) control of spacecraft relative motion, where exists strong kinematic and dynamic couplings between the relative rotation and translation.

Among various mathematical tools for describing the coupled rotation and translation, such as homogeneous transformation matrix [5-7], quaternion or modified Rodrigues parameters plus position vector [8-12], and so on, dual quaternion has shown to be well suitable to represent the six-DOF motion because it not only is a compact, global nonsingular, and computationally efficient parameterization for the rigid body pose but also captures complex couplings between rotation and translation in a natural and implicit way [13-17]. Dual quaternions have already been successfully applied to kinematic analysis in various research fields, such as robot calibration [18], navigation [19], pose estimation [20,21], and inverse kinematics [22].

By virtue of the dual inertial operator introduced by Brodsky and Shoham [23], Wang et al. [24] first derived relative dynamics between two spacecraft using dual quaternions. Replacing the dual inertial operator by an invertible block diagonal matrix, Filipe and Tsiotras [25] rewrote the dual quaternion-based dynamic equation in a more convenient 8-dimensional (8D) matrix/vector form. Based on these two types of governing equations given in [24] or [25], lots of control laws have been proposed to deal with the pose tracking problem under various situations [26-35], among which the unknown disturbance, as an inevitable factor in practice, has been intensively studied in 
recent years. To attenuate small amplitudes of disturbances, proportional-derivative- (PD-) like controllers were derived in [26-28]. However, this robustness against disturbances is at the expense of high proportional gains, which is undesirable. Several sliding mode controllers (SMCs) were proposed in [29-32] to reject general bounded disturbances. However, there is often a trade-off between the chattering suppression and control precision. In [33], the internal model-based control was specially utilized to compensate for sinusoidal and constant disturbances, while the frequencies of all disturbances must be exactly known. Regarding disturbances as unknown parameters, adaptive control laws based on certainty equivalence $(\mathrm{CE})$ principle were designed in $[27,28]$. Since the parameter adaption laws are driven by the tracking errors of the system states, dynamic behaviours of the parameter estimation are unexpected. Besides, even if the corresponding true values are reached, the estimations still keep updating unless the state tracking errors are zero. Thus, the CE-based adaptive control often leads to degradation of the closed-loop performance [36, 37].

As a novel noncertainty equivalent (NCE) method for adaptive control of nonlinear systems with uncertain parameters [38-41], immersion and invariance (I\&I) adaptive control has great potential to overcome many limitations resulting from the CE-based methods. It has been effectively utilized in the attitude [36, 42-45] and/or orbit control [46-49] with unknown inertial or mass parameters and has shown significant improvements in both closed-loop performance and adjustability of the estimation convergence process. However, little attention has been paid to its application in tackling unknown disturbances in the six-DOF motion control.

Motivated by the above observations, this paper investigates the I\&I adaptive pose tracking control of spacecraft subject to general bounded unknown disturbances in the framework of dual quaternions. Similar to [19], the matrix/ vector form of dual number algebraic operations is adopted. However, by introducing a dual direction cosine matrix and a 6D antiblock dual inertial matrix, the swap operations induced by the $8 \mathrm{D}$ diagonal dual inertial matrix in [25] can be avoided, and dimensions of the resultant system equations can be lowered to six. On this basis, an estimation law is designed based on I\&I adaptive control theory to compensate for the general unknown disturbances, which enables the overall adaptive control law to take a modular form. That is, the dynamics of parameter estimations can be adjusted independently of the applied control laws and can realize "parameter lock," which are impossible with the CE-based adaptive control. The overall control scheme is completed by combining the estimated disturbances with a PD-like pose tracking controller. Simulation results verify the effectiveness and benefits of the proposed adaptive control strategy when compared with a CEbased adaptive controller and a SMC.

The rest of the paper is organized as follows. Section 2 introduces necessary preliminaries of quaternions and dual quaternions. On this basis, the dual quaternion-based model of relative motion between two spacecraft written in 6D vector/ matrix form is derived in Section 3. In Section 4, firstly the I\&I- based adaptive disturbance estimation law is derived and then the complete adaptive pose tracking controller is designed along with Lyapunov stability proof. Simulation and comparison results are presented in Section 5 to demonstrate the features and effectiveness of the proposed adaptive control scheme. Section 6 concludes this paper.

\section{Preliminaries}

2.1. Quaternions. A quaternion is defined as $\mathrm{q}=\left[\begin{array}{ll}q_{0} & \mathbf{q}^{T}\end{array}\right]^{T}$ $\in H$, where $q_{0} \in R$ and $\mathbf{q} \in R^{3}$ are its scalar and vector parts. Vectors can be viewed as vector quaternions which are quaternions with zero scalar parts and vice versa. $\mathrm{q}^{*}=\left[q_{0}-\mathbf{q}^{T}\right]^{T}$ is the conjugate of $\mathrm{q} \cdot 1_{H}=\left[\begin{array}{ll}1 & \mathbf{0}^{T}\end{array}\right]^{T}$ and $0_{H}=\left[\begin{array}{ll}0 & \mathbf{0}^{T}\end{array}\right]^{T}$ are unit and zero quaternions where $\mathbf{0}=\left[\begin{array}{lll}0 & 0 & 0\end{array}\right]^{T}$. A unit quaternion satisfies $\mathrm{qq}^{*}=1_{H}$. For any two quaternions $\mathrm{a}=\left[\begin{array}{ll}a_{0} & \mathbf{a}^{T}\end{array}\right]^{T}$ and $\mathrm{b}=\left[\begin{array}{ll}b_{0} & \mathbf{b}^{T}\end{array}\right]^{T}$, some necessary operations are given as follows:

$$
\begin{aligned}
& \text { multiplication: } \mathrm{ab}=\left[\begin{array}{c}
a_{0} b_{0}+\mathbf{a} \cdot \mathbf{b} \\
a_{0} \mathbf{b}+b_{0} \mathbf{a}+\mathbf{a} \times \mathbf{b}
\end{array}\right], \\
& \text { dot product: } \mathrm{a} \cdot \mathrm{b}=\mathrm{a}^{T} \mathrm{~b}=a_{0} b_{0}+\mathbf{a} \cdot \mathbf{b}, \\
& \text { vector part: } \operatorname{vec}(\mathrm{a})=\mathbf{a} .
\end{aligned}
$$

If the attitude of frame $\mathscr{F}_{X}$ w.r.t. $\mathscr{F}_{Y}$ is represented by the unit quaternion $\mathrm{q}_{X Y}$, then the coordinate transform of a vector $\mathbf{r}$ is given as $\mathrm{r}^{X}=\mathrm{q}_{X Y}^{*} \mathrm{r}^{Y} \mathrm{q}_{X Y}$, where $\mathrm{r}=\left[\begin{array}{ll}0 & \mathbf{r}^{T}\end{array}\right]^{T}$ is the quaternion form of $\mathbf{r}$ and is used to match the dimensions of the quaternion and vector. The $3 \mathrm{D}$ form coordinate transform can be given as

$$
\mathbf{r}^{X}=\mathbf{C}_{X Y} \mathbf{r}^{Y}=\mathbf{T}\left(\mathrm{q}_{X Y}, \mathrm{q}_{X Y}\right) \mathbf{r}^{Y}
$$

where $\mathbf{C}_{X Y}$ is the direction cosine matrix (DCM); $\mathbf{T}(\cdot, \cdot): H \times H \longrightarrow R^{3 \times 3}$ is defined as

$$
\mathbf{T}(\mathrm{a}, \mathrm{b})=\mathbf{a b}^{T}+\left(a_{0} \mathbf{I}_{3}-\mathbf{a}^{\times}\right)\left(b_{0} \mathbf{I}_{3}-\mathbf{b}^{\times}\right) .
$$

2.2. Dual Numbers and Dual Vectors. A dual number is defined as $\widehat{a}=a_{r}+\epsilon a_{d} \in \widehat{R}$, where $a_{r}, a_{d} \in R$ are the real and dual parts. $\epsilon$ is the dual unit and satisfies $\varepsilon^{2}=0, \varepsilon \neq 0$. When real and dual parts are both vectors, a dual number is extended to a dual vector with a form $\widehat{\mathbf{a}}=\mathbf{a}_{r}+\varepsilon \mathbf{a}_{d} \in \widehat{R}^{3}$ where $\mathbf{a}_{r}, \mathbf{a}_{d} \in R^{3}$. The zero dual vector is denoted by $\widehat{\mathbf{0}}=\mathbf{0}+\varepsilon \mathbf{0}$. Since $\widehat{R}^{3}$ is isomorphic to $R^{6}$ [21], a dual vector can be equivalently denoted as a vector form $\widehat{\mathbf{a}}=\left[\mathbf{a}_{r}^{T} \mathbf{a}_{d}^{T}\right]^{T}$. For any $\widehat{\mathbf{a}}, \widehat{\mathbf{b}} \in \widehat{R}^{3}$, some important operations are defined as follows:

$$
\begin{aligned}
& \text { Swap: } \widehat{\mathrm{a}}^{\mathrm{s}}=\mathbf{a}_{d}+\varepsilon \mathbf{a}_{r}, \\
& \text { Corresponding product: } \widehat{a} \odot \widehat{\mathbf{a}}=a_{r} \mathbf{a}_{r}+\varepsilon a_{d} \mathbf{a}_{d}, \\
& \text { Cross product: } \widehat{\mathbf{a}} \times \widehat{\mathbf{b}}=\mathbf{a}_{r} \times \mathbf{b}_{r}+\varepsilon\left(\mathbf{a}_{r} \times \mathbf{b}_{d}+\mathbf{a}_{d} \times \mathbf{b}_{r}\right) \text {, } \\
& \text { Circle product: } \widehat{\mathbf{a}} \circ \widehat{\mathbf{b}}=\mathbf{a}_{r} \cdot \mathbf{b}_{r}+\mathbf{a}_{d} \cdot \mathbf{b}_{d}, \\
& \text { Norm: }\|\widehat{\mathbf{a}}\|=\sqrt{\widehat{\mathbf{a}} \circ \widehat{\mathbf{a}}}
\end{aligned}
$$


Based on the above definitions, the following properties can be obtained:

$$
\begin{aligned}
\widehat{\mathbf{a}} \circ(\widehat{\mathbf{b}} \times \widehat{\mathbf{c}}) & =\widehat{\mathbf{b}}^{s} \circ\left(\widehat{\mathbf{c}} \times \widehat{\mathbf{a}}^{s}\right)=\widehat{\mathbf{c}}^{s} \circ\left(\widehat{\mathbf{a}}^{s} \times \widehat{\mathbf{b}}\right), \\
\widehat{\mathbf{a}}^{s} \circ \widehat{\mathbf{b}}^{s} & =\widehat{\mathbf{a}} \circ \widehat{\mathbf{b}}=\widehat{\mathbf{b}} \circ \widehat{\mathbf{a}}, \\
\widehat{\mathbf{a}} \times \widehat{\mathbf{a}} & =\widehat{0} .
\end{aligned}
$$

2.3. Dual Quaternions. A dual quaternion is defined as $\widehat{\mathrm{q}}=\mathrm{q}_{r}+\varepsilon \mathrm{q}_{d} \in \widehat{H}$, with $\mathrm{q}_{r}, \mathrm{q}_{d} \in H$. Dual quaternions composed of vector quaternions can be viewed as dual vectors and vice versa. $\widehat{H}$ is isomorphic to $R^{8}$, and thus $\widehat{\mathrm{q}}$ can also be denoted as $\widehat{\mathrm{q}}=\left[\begin{array}{ll}\mathrm{q}_{r}^{T} & \mathrm{q}_{d}^{T}\end{array}\right]^{T} \cdot \widehat{\mathrm{q}}^{*}=\mathrm{q}_{r}^{*}+\varepsilon \mathrm{q}_{d}^{*}$ is the conjugate $\widehat{\mathrm{q}}$. $\widehat{1}=1+\varepsilon 0$ is the unit dual quaternion. Unit dual quaternions satisfy $\widehat{\mathrm{q}} \widehat{\mathrm{q}}^{*}=\widehat{1}$. Apart from the cross product, operations given in (4) are still valid for dual quaternions $\widehat{a}$ and $\widehat{b}$. Additionally, there are

$$
\begin{aligned}
& \text { multiplication : } \widehat{a} \widehat{b}=\mathrm{a}_{r} \mathrm{~b}_{r}+\varepsilon\left(\mathrm{a}_{r} \mathrm{~b}_{d}+\mathrm{a}_{d} \mathrm{~b}_{r}\right), \\
& \operatorname{vector} \operatorname{part}: \operatorname{vec}(\widehat{\mathrm{a}})=\widehat{\mathbf{a}}=\mathbf{a}_{r}+\varepsilon \mathbf{a}_{d} .
\end{aligned}
$$

Besides, there are the following properties:

$$
\begin{aligned}
\widehat{a} \circ \widehat{b} & =\widehat{\mathbf{a}} \circ \operatorname{vec}(\widehat{b}), \\
\widehat{a} \circ(\widehat{b} \widehat{c}) & =\widehat{b}^{s} \circ\left(\widehat{a}^{s} \widehat{c}^{*}\right)=\widehat{c}^{s}\left(\widehat{b}^{*} \widehat{a}^{s}\right) .
\end{aligned}
$$

Supposing the unit quaternion $\widehat{\mathrm{q}}_{X Y}$ represents the pose of $\mathscr{F}_{X}$ w.r.t. $\mathscr{F}_{Y}$, then the coordinate transform of a dual vector $\widehat{r}$ is given by $\widehat{\mathrm{r}}^{X}=\widehat{\mathrm{q}}_{X Y}^{*} \widehat{r}^{Y} \widehat{\mathrm{q}}_{X Y}$, which is written in a $8 \mathrm{D}$ form to match the dimensions of the dual quaternion and dual vector. By introducing the dual direction cosine matrix (DDCM) $\widehat{\mathbf{C}}_{X Y}$, which is a counterpart of $\mathbf{C}_{X Y}$ in dual form, the $6 \mathrm{D}$ form coordinate transform can be given as

$$
\widehat{\mathbf{r}}^{X}=\widehat{\mathbf{C}}_{X Y} \widehat{\mathbf{r}}^{Y}=\widehat{T}\left(\widehat{\mathrm{q}}_{X Y}\right) \widehat{\mathbf{r}}^{Y},
$$

where $\widehat{T}(\cdot): \hat{H} \longrightarrow R^{6 \times 6} \quad$ is defined as $\widehat{\mathrm{T}}(\widehat{\mathrm{q}})$ $=\left[\begin{array}{cc}\mathrm{T}\left(\mathrm{q}_{r}, \mathrm{q}_{r}\right) & \mathbf{0}_{3 \times 3} \\ 2 \mathrm{~T}\left(\mathrm{q}_{r}, \mathrm{q}_{d}\right) & \mathrm{T}\left(\mathrm{q}_{r}, \mathrm{q}_{r}\right)\end{array}\right]$.

\section{Spacecraft Dynamics Based on Dual Quaternions}

The following frames are needed: $\mathscr{F}_{I}$ is the Earth centred inertial frame; $\mathscr{F}_{B}$ and $\mathscr{F}_{T}$ denote the body fixed frame of the chaser and target spacecraft, respectively, and their origins locate at the center of mass (c.m.) of the corresponding spacecraft and axes point along the principal axes of inertias; $\mathscr{F}_{O}$ is the orbit frame, whose origin is at c.m. of the spacecraft, $x_{O}$ axis points along the radius direction, and $z_{\mathrm{O}}$ is normal to the orbit plane. Hereafter, the superscript of a quantity denotes the frame in which it is expressed.

If the attitude and displacement of $\mathscr{F}_{B}$ w.r.t. $\mathscr{F}_{I}$ are represented by $\mathrm{q}_{B I}$ and vector $\mathbf{t}_{B I}$, then the pose of $\mathscr{F}_{B}$ w.r.t. $\mathscr{F}_{I}$ can be described as a unit dual quaternion as follows:

$$
\widehat{\mathrm{q}}_{B I}=\mathrm{q}_{B I}+\varepsilon \frac{\mathrm{q}_{B I} \mathrm{t}_{B I}^{B}}{2},
$$

where $t_{B I}^{B}=\left[\begin{array}{ll}0 & \left(t_{B I}^{B}\right)^{\mathrm{T}}\end{array}\right]^{\mathrm{T}}$. Taking derivative of (9), the kinematic equation of a rigid body in terms of dual quaternion is obtained as

$$
\dot{\hat{\mathrm{q}}}_{B I}=\frac{\widehat{\mathrm{q}}_{B I} \widehat{\omega}_{B I}^{B}}{2},
$$

where $\widehat{\omega}_{B I}^{B}$ is the dual quaternion form of dual velocity $\widehat{\boldsymbol{\omega}}_{B I}^{B}=\boldsymbol{\omega}_{B I}^{B}+\varepsilon \mathbf{v}_{B I}^{B}, \boldsymbol{\omega}_{B I}^{B}$ is the angular velocity, and $\mathbf{v}_{B I}^{B}=\dot{\mathbf{t}}_{B I}^{B}+$ $\boldsymbol{\omega}_{B I}^{B} \times \mathbf{t}_{B I}^{B}$ is the linear velocity. By replacing $\mathscr{F}_{B}$ with $\mathscr{F}_{T}$, kinematic equation of the target spacecraft can be described in the same way.

To describe the six-DOF dynamics of the spacecraft in a $6 \mathrm{D}$ vector/matrix form, instead of using the $8 \times 8$ diagonal dual inertial matrix given in [25], the novel $6 \times 6$ antidiagonal dual inertial matrix is defined as

$$
\widehat{\mathbf{M}}_{B}=\left[\begin{array}{cc}
\mathbf{0}_{3 \times 3} & m_{B} \mathbf{I}_{3} \\
\mathbf{J}_{B} & \mathbf{0}_{3 \times 3}
\end{array}\right] \text {, }
$$

where $m_{B}$ and $\mathbf{J}_{B}$ are the mass and inertial matrix of the chaser spacecraft. By virtue of (11), the dynamic equation of the spacecraft can be given as

$$
\widehat{\mathbf{M}}_{B} \dot{\widehat{\boldsymbol{\omega}}}_{B I}^{B}+\widehat{\boldsymbol{\omega}}_{B I}^{B} \times \widehat{\mathbf{M}}_{B} \widehat{\boldsymbol{\omega}}_{B I}^{B}=\widehat{\mathbf{f}}^{B}
$$

where $\widehat{\mathbf{f}}^{B}=\mathbf{f}^{B}+\varepsilon \tau^{B}$ is the total external dual force exerted on the spacecraft and $\mathbf{f}^{B}$ and $\tau^{B}$ are the corresponding force and torque.

The relative pose between $\mathscr{F}_{B}$ and $\mathscr{F}_{T}$ is calculated by

$$
\widehat{\mathrm{q}}_{B T}=\widehat{\mathrm{q}}_{T}^{*} \widehat{\mathrm{q}}_{B}=\mathrm{q}_{B T}+\varepsilon \frac{1}{2} \mathrm{q}_{B T} \mathrm{t}_{B T}^{B} \text {. }
$$

Differentiating (13), the relative kinematics can be obtained as

$$
\dot{\widehat{\mathrm{q}}}_{B T}=\frac{\widehat{\mathrm{q}}_{B T} \widehat{\omega}_{B T}^{B}}{2},
$$

where $\widehat{\omega}_{B T}^{B}=\widehat{\omega}_{B I}^{B}-\widehat{\mathrm{q}}_{B T}^{*} \widehat{\omega}_{T I}^{T} \widehat{\mathrm{q}}_{B T}$ is the relative dual velocity in dual quaternion form. By using the DDCM defined in (8), the $6 \mathrm{D}$ form of relative dual velocity can be calculated by

$$
\widehat{\boldsymbol{\omega}}_{B T}^{B}=\widehat{\boldsymbol{\omega}}_{B I}^{B}-\widehat{\mathbf{C}}_{B T} \widehat{\boldsymbol{\omega}}_{T I}^{T} \text {. }
$$

Substituting (15) into (12), the dual quaternion-based relative dynamics written in $6 \mathrm{D}$ form can be obtained as

$$
\widehat{\mathbf{M}}_{B} \dot{\hat{\boldsymbol{\omega}}}_{B T}^{B}=-\widehat{\boldsymbol{\omega}}_{B I}^{B} \times \widehat{\mathbf{M}}_{B} \widehat{\boldsymbol{\omega}}_{B I}^{B}-\widehat{\mathbf{M}}_{B}\left(\widehat{\mathbf{C}}_{B T} \dot{\hat{\boldsymbol{\omega}}}_{T I}^{T}-\widehat{\boldsymbol{\omega}}_{B T}^{B} \times \widehat{\boldsymbol{\omega}}_{T I}^{T}\right)+\widehat{\mathbf{f}}^{B} .
$$

Besides, (16) can be further expressed as

$$
\widehat{\mathbf{M}}_{B} \dot{\hat{\boldsymbol{\omega}}}_{B T}^{B}=\widehat{\mathbf{S}} \widehat{\boldsymbol{\omega}}_{B I}^{B}-\widehat{\mathbf{M}}_{B} \widehat{\mathbf{C}}_{B T} \dot{\hat{\boldsymbol{\omega}}}_{T I}^{T}-\widehat{\boldsymbol{\omega}}_{T I}^{B} \times \widehat{\mathbf{M}}_{B} \widehat{\boldsymbol{\omega}}_{T I}^{B}+\widehat{\mathbf{f}}^{B},
$$

where $\widehat{\mathbf{S}}=\left[\begin{array}{cc}\mathbf{0}_{3 \times 3} & \mathbf{S}_{1} \\ \mathbf{S}_{2} & \mathbf{0}_{3 \times 3}\end{array}\right]$ and $\mathbf{S}_{1}$ and $\mathbf{S}_{2}$ are skew-symmetric matrices which are given by

$$
\begin{aligned}
& \mathbf{S}_{1}=-m_{B}\left(\boldsymbol{\omega}_{B T}^{B}+2 \boldsymbol{\omega}_{T I}^{B}\right)^{\times} \\
& \mathbf{S}_{2}=\left[\mathbf{J}_{B}\left(\boldsymbol{\omega}_{B T}^{B}+\boldsymbol{\omega}_{T I}^{B}\right)\right]^{\times}-\left(\boldsymbol{\omega}_{B I}^{B}\right)^{\times} \mathbf{J}_{B}-\mathbf{J}_{B}\left(\boldsymbol{\omega}_{T I}^{B}\right)^{\times} .
\end{aligned}
$$


Remark 1. Compared to the $8 \mathrm{D}$ dynamic equations given in [25], by virtue of the novel dual inertial matrix defined in (11) and the DDCM defined in (8), the dynamic equations given in (13) and (16) avoid swap operation on the dual velocity induced by the diagonal dual inertial matrix given in [25], and their dimensions are lowered to six.

For a spacecraft that orbits the Earth, $\mathbf{f}^{B}$ can be expressed as

$$
\widehat{\mathbf{f}}^{B}=\widehat{\mathbf{f}}_{u}^{B}+\widehat{\mathbf{f}}_{g}^{B}+\widehat{\mathbf{f}}_{\nabla \boldsymbol{g}}^{B}+\widehat{\mathbf{f}}_{J_{2}}^{B}+\widehat{\mathbf{f}}_{d}^{B}
$$

where $\widehat{\mathbf{f}}_{u}^{B}=\mathbf{f}_{u}^{B}+\epsilon \tau_{u}^{B}$ is the dual control force and $\widehat{\mathbf{f}}_{g}^{B}, \widehat{\mathbf{f}}_{\nabla g}^{B}$, and $\widehat{\mathbf{f}}_{J_{2}}^{B}$ are gravitational force, gravity gradient torque, and oblateness perturbation force written in dual form and can be calculated by

$$
\begin{aligned}
\widehat{\mathbf{f}}_{g}^{B} & =\widehat{\mathbf{M}}_{B} \widehat{\mathbf{a}}_{g}^{B}, \\
\widehat{\mathbf{f}}_{\nabla \boldsymbol{g}}^{B} & =3 \mu \frac{\widehat{\mathbf{t}}_{B I}^{B} \times \mathbf{M}_{b} \widehat{\mathbf{t}}_{B I}^{B}}{\left\|\hat{\mathbf{t}}_{B I}^{B}\right\|^{5}}, \\
\widehat{\mathbf{f}}_{J_{2}}^{B} & =\widehat{\mathbf{M}}_{B} \widehat{\mathbf{a}}_{J_{2}}^{B},
\end{aligned}
$$

where $\mu=398600.4418\left(\mathrm{~km}^{3} / \mathrm{s}^{2}\right)$ is the Earth's gravitational parameter; $\widehat{\mathbf{a}}_{g}^{B}=\mathbf{0}+\varepsilon \mathbf{a}_{g}^{B}, \widehat{\mathbf{a}}_{J_{2}}^{B}=\mathbf{0}+\varepsilon \mathbf{a}_{J_{2}}^{B}$, and $\widehat{\mathbf{t}}_{B I}^{B}=\mathbf{t}_{B I}^{B}+\varepsilon \mathbf{0}$; gravitational and $J_{2}$ accelerations $\mathbf{a}_{g}^{B}$ and $\mathbf{a}_{J 2}^{B}$ are given as

$$
\begin{aligned}
& \mathbf{a}_{g}^{B}=-\mu \frac{\mathbf{t}_{B I}^{B}}{\left\|\mathbf{t}_{B I}^{B}\right\|^{3}}, \\
& \mathbf{a}_{J_{2}}^{B}=-\mathbf{C}_{B I} \frac{3}{2} \frac{\mu J_{2} R_{e}^{2}}{\left\|\mathbf{t}_{B I}^{I}\right\|^{5}}\left[\mathbf{D}-5\left(\frac{t_{B I}^{z}}{\mathbf{t}_{B I}^{I}}\right)^{2} \mathbf{I}_{3}\right] \mathbf{t}_{B I}^{I},
\end{aligned}
$$

where $R_{e}=6378.137 \mathrm{kmis}$ the Earth's mean equatorial radius; $J_{2}=0.0010826267 ; \mathbf{D}=\operatorname{diag}(1,1,3) ; t_{B I}^{z}$ is the third component of $\mathbf{t}_{B I}^{I}$; and $\mathbf{C}_{B I}$ is the DCM.

$\widehat{\mathbf{f}}_{d}^{B}=\mathbf{f}_{d}^{B}+\varepsilon \tau_{d}^{B}$ is the time-varying dual disturbance force due to atmosphere drag, solar radiation, third bodies, and so on. It is assumed that dual disturbance and its first derivative are bounded. Note that a dual number (vector or quaternion) is bounded if and only if its real and dual parts are both bounded.

\section{I\&I Adaptive Control Law Design}

The pose tracking control of spacecraft subject to unknown disturbances is considered in this paper. Designed based on the I\&I adaptive control theory, the overall control strategy takes a modular structure which includes a disturbance estimator and a pose tracking control law, designed separately.

4.1. Disturbance Estimator. Treating disturbances $\widehat{\mathbf{f}}_{d}^{B}$ as unknown parameters, based on the I\&I adaptive control theory, the disturbance estimator $\underline{\widehat{\mathbf{f}}}_{d}^{B}$ has the following form:

$$
\widehat{\mathbf{f}}_{d}^{B}=\widehat{\boldsymbol{\xi}}+\widehat{\boldsymbol{\beta}}\left(\widehat{\mathbf{M}}_{B} \widehat{\boldsymbol{\omega}}_{B I}^{B}\right),
$$

where $\widehat{\xi}=\widehat{\xi}_{f}+\epsilon \widehat{\xi}_{\tau}$ is the dynamic part of the estimation and $\widehat{\boldsymbol{\beta}}\left(\widehat{\mathbf{M}}_{B} \widehat{\boldsymbol{\omega}}_{B I}^{B}\right)$ is a continuous vector function to be specified.

The disturbance estimation error is defined as

$$
\widehat{\mathbf{f}}_{d}^{B}=\widehat{\mathbf{f}}_{d}^{B}-\widehat{\mathbf{f}}_{d}^{B}=\widehat{\boldsymbol{\xi}}+\widehat{\boldsymbol{\beta}}\left(\widehat{\mathbf{M}}_{B} \widehat{\boldsymbol{\omega}}_{B I}^{B}\right)-\widehat{\mathbf{f}}_{d}^{B} .
$$

Combined with (12), the time derivative of (23) can be obtained as

$$
\hat{\mathbf{f}}_{d}^{B}=\dot{\hat{\boldsymbol{\xi}}}+\frac{\partial \hat{\boldsymbol{\beta}}}{\partial \hat{\mathbf{M}}_{B} \hat{\boldsymbol{\omega}}_{B I}^{B}}-\hat{\boldsymbol{\omega}}_{B I}^{B} \times \hat{\mathbf{M}}_{B} \hat{\boldsymbol{\omega}}_{B I}^{B}+\hat{\mathbf{f}}^{B}-\dot{\hat{\mathbf{f}}}_{d}^{B} .
$$

Based on the observation of (24), the update law of $\widehat{\xi}$ can be selected as

$$
\dot{\overrightarrow{\boldsymbol{\xi}}}=-\frac{\partial \widehat{\boldsymbol{\beta}}}{\partial\left(\widehat{\mathbf{M}}_{B} \widehat{\boldsymbol{\omega}}_{B I}^{B}\right)}\left(-\hat{\boldsymbol{\omega}}_{B I}^{B} \times \widehat{\mathbf{M}}_{B} \widehat{\boldsymbol{\omega}}_{B I}^{B}+\widehat{\mathbf{f}}_{u}^{B}+\widehat{\mathbf{f}}_{g}^{B}+\widehat{\mathbf{f}}_{J_{2}}^{B}+\widehat{\mathbf{f}}_{\nabla \boldsymbol{g}}^{B}+\widehat{\mathbf{f}}_{d}^{B}\right) .
$$

Substituting (25) into (24) yields

$$
\hat{\mathbf{f}}_{d}^{B}=\frac{\partial \hat{\boldsymbol{\beta}}}{\partial \hat{\mathbf{M}}_{B} \hat{\boldsymbol{\omega}}_{B I}^{B}} \mathbf{f}-\dot{\hat{\mathbf{f}}}_{d}^{B} .
$$

To stabilize the subsystem of estimation error, the function $\widehat{\boldsymbol{\beta}}$ can be chosen as

$$
\widehat{\boldsymbol{\beta}}=-\lambda\left(\widehat{\mathbf{M}}_{B} \widehat{\boldsymbol{\omega}}_{B I}^{B}\right),
$$

with $\lambda>0$. Substituting (27) into (26), we get

$$
\dot{\mathbf{f}}_{d}^{B}=-\lambda \mathbf{f}_{d}^{B}-\dot{\hat{\mathbf{f}}}_{d}^{B} .
$$

Consider the Lyapunov candidate $V_{d}=\left(\left(\underset{\widetilde{\mathbf{f}}}{\widehat{d}} d^{B} \circ \underset{\sim}{\widehat{\mathbf{f}}} \underset{d}{B}\right) / 2\right)$. Its time derivative along (28) can be obtained as

$$
\dot{V}_{d}=\hat{\mathbf{f}}_{d}^{B} \circ\left(-\lambda \hat{\mathbf{f}}_{d}^{B}+\dot{\hat{\mathbf{f}}}_{d}^{B}\right) \leq-\left\|\hat{\mathbf{f}}_{d}^{B}\right\|\left(\lambda\left\|\widehat{\mathbf{f}}_{d}^{B}\right\|-\left\|\dot{\hat{\mathbf{f}}}_{d}^{B}\right\|\right) .
$$

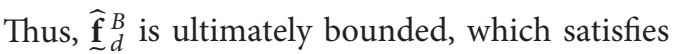

$$
\lim _{t \rightarrow \infty}\left\|\widehat{\hat{f}}_{d}^{B}\right\| \leq \frac{\left\|\dot{\overrightarrow{\mathbf{f}}}_{d}^{B}\right\|}{\lambda} .
$$

Since $\dot{\hat{\mathbf{f}}}_{d}^{B}$ is bounded, the ultimate bound of disturbance estimation error can be made arbitrarily small by choosing large enough $\lambda$. When the disturbances are constant, there is

$$
\dot{V}_{d} \leq-2 \lambda V_{d}
$$

It can be readily obtained from (31) that ${\underset{\sim}{\mathbf{f}}}_{d}^{B}$ is exponentially convergent. In summary, the disturbance estimator designed based on the I\&I adaptive method is given as 


$$
\left\{\underline{\mathbf{f}}_{d}^{B}=\widehat{\boldsymbol{\xi}}+\lambda\left(\widehat{\mathbf{M}}_{B} \widehat{\boldsymbol{\omega}}_{B I}^{B}\right), \dot{\vec{\xi}}=-\lambda\left(-\widehat{\boldsymbol{\omega}}_{B I}^{B} \times \widehat{\mathbf{M}}_{B} \widehat{\boldsymbol{\omega}}_{B I}^{B}+\widehat{\mathbf{f}}_{u}^{B}+\widehat{\mathbf{f}}_{g}^{B}+\widehat{\mathbf{f}}_{\nabla \boldsymbol{g}}^{B}+\widehat{\mathbf{f}}_{J_{2}}^{B}+\widehat{\mathbf{f}}_{d}^{B}\right) .\right.
$$

Remark 2. According the I\&I adaptive control theory, the designs of disturbance estimation and pose tracking law are separate. Thus, the proposed disturbance estimator (32) can be combined with different control laws.
4.2. Pose Tracking Control Law. Combined with the disturbance estimation given by (32), the PD-like adaptive pose tracking control law is designed as

$$
\widehat{\mathbf{f}}_{u}^{B}=-\left(\widehat{k}_{1} \odot \widehat{\mathbf{p}}_{B T}^{B}\right)^{s}-\left(\widehat{k}_{2} \odot \widehat{\boldsymbol{\omega}}_{B T}^{B}\right)^{s}+\widehat{\boldsymbol{\omega}}_{T I}^{B} \times \widehat{\mathbf{M}}_{B} \widehat{\boldsymbol{\omega}}_{T I}^{B}+\widehat{\mathbf{M}}_{B} \widehat{\mathbf{C}}_{B T} \dot{\boldsymbol{\mathbf { \omega }}}_{T I}^{T}-\widehat{\mathbf{M}}_{B} \widehat{\mathbf{a}}_{g}^{B}-3 \mu \frac{\widehat{\mathbf{t}}_{B I}^{B} \times \mathbf{M}_{B} \hat{\mathbf{t}}_{B I}^{B}}{\left\|\hat{\mathbf{t}}_{B I}^{B}\right\|^{5}} \widehat{\mathbf{M}}_{B} \widehat{\mathbf{a}}_{J_{2}}^{B}+\widehat{\mathbf{f}}_{d}^{B},
$$

where $\widehat{k}_{1}=k_{q}+\varepsilon 2 k_{t}$ and $\widehat{k}_{2}=k_{\boldsymbol{\omega}}+\varepsilon k_{v}$ with $k_{q}, k_{t}, k_{\boldsymbol{\omega}}, k_{v}>0$, and $\hat{\mathbf{p}}_{B T}^{B}$ is calculated by

$$
\widehat{\mathbf{p}}_{B T}^{B}=\operatorname{vec}\left(\left(\widehat{\mathrm{q}}_{B T}^{*}\left(\widehat{\mathrm{q}}_{B T}-\widehat{1}_{H}\right)^{s}\right)^{s}\right)=\mathbf{q}_{B T}+\varepsilon \frac{\mathbf{t}_{B T}^{B}}{2} \in \widehat{R}^{3} .
$$

Remark 3. $\mathbf{q}_{B T}$ is the vector part of relative attitude quaternion $\mathbf{q}_{B T}$, and $\mathbf{t}_{B T}^{B}$ is the relative position vector; therefore, $\widehat{\mathbf{p}}_{B T}^{B}$ can also reflect the pose tracking error.

The relationships between $\widehat{\mathbf{p}}_{B T}^{B}, \widehat{\mathrm{q}}_{B T}^{B}$, and $\widehat{\boldsymbol{\omega}}_{B T}^{B}$ are given in the following lemmas.

Lemma 1. For any $\widehat{a}=a_{r}+\varepsilon a_{d}$, there is $\left[\hat{a} \odot\left(\widehat{\mathbf{q}}_{B T}-\widehat{1}_{H}\right)\right] \circ$ $\left(\widehat{\mathbf{q}}_{B T}-\widehat{1}_{H}\right) \geq\left(\widehat{a} \odot \widehat{\mathbf{p}}_{B T}^{B}\right) \circ \widehat{\mathbf{p}}_{B T}^{B}$.

Proof. According to the definition of circle product in (4), Lemma 1 can be easily verified, which is omitted here.

Lemma 2. For any $\widehat{a}=a_{r}+\varepsilon a_{d}$, there is $\left(\widehat{a} \odot\left(\widehat{\mathbf{q}}_{B T}-\widehat{\mathbf{1}}_{H}\right)\right)$ 。 $\left(\widehat{\mathbf{q}}_{B T} \widehat{\boldsymbol{\omega}}_{B T}^{B}\right)=\widehat{\boldsymbol{\omega}}_{B T}^{B} \circ\left(\widehat{a} \odot \widehat{\mathbf{p}}_{B T}^{B}\right)$.
Proof. Applying properties given in (4) and (7), there is ( $\widehat{a} \odot$ $\left.\left(\widehat{\mathbf{q}}_{B T}-\widehat{\mathbf{1}}_{H}\right)\right) \circ\left(\widehat{\mathbf{q}}_{B T} \widehat{\boldsymbol{\omega}}_{B T}^{B}\right)=\left(\widehat{\boldsymbol{\omega}}_{B T}^{B}\right)^{s} \circ \operatorname{vec}\left[\widehat{\mathbf{q}}_{B T}^{*}\left(\widehat{\mathbf{\mathbf { a }}} \odot\left(\widehat{\mathbf{q}}_{B T}-\widehat{\mathbf{1}}_{H}\right)\right)^{\mathrm{s}}\right]=$ $\left(\widehat{\boldsymbol{\omega}}_{B T}^{B}\right)^{s} \circ \operatorname{vec}\left[\left(a_{d} \mathbf{t}_{B T}^{B} / 2\right)+\varepsilon a_{r}\left(\mathbf{1}_{H}-\mathbf{q}_{B T}^{*}\right)\right]=\widehat{\boldsymbol{\omega}}_{B T}^{B} \circ\left(\widehat{a} \odot \widehat{\mathbf{p}}_{B T}^{B}\right)$.

When the disturbances are time-varying, the first result of this paper is presented in the following theorem.

Theorem 1. Consider the closed-loop system composed of relative motion models (14) and (16), disturbance estimator (32), and control law (33). The tracking error states $\widehat{\mathbf{p}}_{B T}^{B}$ and $\widehat{\boldsymbol{\omega}}_{B T}^{B}$ converge to arbitrarily small neighbourhood of the origin by choosing large enough $\lambda$.

Proof. Consider the following candidate Lyapunov function:

$$
\begin{aligned}
V_{1}= & \frac{1}{2}\left(\widehat{\boldsymbol{\omega}}_{B T}^{B}\right)^{s} \circ\left(\widehat{\mathbf{M}}_{B} \widehat{\boldsymbol{\omega}}_{B T}^{B}\right)+\left[\hat{k} \odot\left(\widehat{\mathbf{q}}_{B T}-\widehat{1}_{H}\right)\right] \\
& \circ\left(\widehat{\mathbf{q}}_{B T}-\widehat{1}_{H}\right)+\left(\widehat{c} \odot \widehat{\mathbf{p}}_{B T}^{B}\right)^{s} \circ\left(\widehat{\mathbf{M}}_{B} \widehat{\boldsymbol{\omega}}_{B T}^{B}\right),
\end{aligned}
$$

where $\widehat{k}=\widehat{k}_{1}+\widehat{c} \odot \widehat{k}_{2}, \widehat{c}=c_{\boldsymbol{\omega}}+\varepsilon 2 c_{v}$, and $c_{\boldsymbol{\omega}}$ and $c_{v}$ are small positive constants. To show the positive definiteness of $V$, with Lemma 1, it can be obtained that

$$
2 V_{1} \geq 2\left(k_{q}+c_{\boldsymbol{\omega}} k_{\boldsymbol{\omega}}\right)\left\|\mathbf{q}_{B T}\right\|^{2}+\gamma_{J}\left\|\boldsymbol{\omega}_{B T}^{B}\right\|^{2}+\left(k_{r}+c_{v} k_{v}\right)\left\|\mathbf{t}_{B T}^{B}\right\|^{2}+m_{B}\left\|\mathbf{v}_{B T}^{B}\right\|^{2}-2 c_{\boldsymbol{\omega}} \gamma_{J}\left\|\mathbf{q}_{B T}\right\|\left\|\boldsymbol{\omega}_{B T}^{B}\right\|-2 c_{v} m_{B}\left\|\mathbf{t}_{B T}^{B}\right\|\left\|\mathbf{v}_{B T}^{B}\right\|=\mathbf{x}^{\mathrm{T}} \mathbf{A} \mathbf{x}
$$

where $\gamma_{\boldsymbol{\omega}}=\left\|\boldsymbol{\omega}_{T I}^{B}\right\|$ is the upper bound of the target's angular velocity that is unknown, $\gamma_{J}=\left\|\mathbf{J}_{B}\right\|, \mathbf{x}=\left[\left\|\mathbf{q}_{B T}\right\|\left\|\boldsymbol{\omega}_{B T}^{B}\right\|\left\|\mathbf{t}_{B T}^{B}\right\|\right.$ $\left.\left\|\mathbf{v}_{B T}^{B}\right\|\right]^{\mathrm{T}}$, and

$$
\mathbf{A}=\left[\begin{array}{cccc}
2\left(k_{q}+c_{\boldsymbol{\omega}} k_{\boldsymbol{\omega}}\right) & -c_{\boldsymbol{\omega}} \gamma_{J} & 0 & 0 \\
-c_{\boldsymbol{\omega}} \gamma_{J} & \gamma_{J} & 0 & 0 \\
0 & 0 & k_{r}+c_{v} k_{v} & -c_{v} m_{B} \\
0 & 0 & -c_{v} m_{B} & m_{B}
\end{array}\right]
$$


It can be easily verified that the positive definiteness of $\mathbf{A}$ can be guaranteed by small enough $c_{\boldsymbol{\omega}}$ and $c_{v}$. Applying Lemma 2, the time derivative of $V$ can be given as

$$
\begin{aligned}
\dot{V}_{1}= & \left(\widehat{\boldsymbol{\omega}}_{B T}^{B}\right)^{s} \circ\left[\widehat{\mathbf{S}} \widehat{\boldsymbol{\omega}}_{B T}^{B}-\left(\widehat{k}_{1} \odot \widehat{\mathbf{p}}_{B T}^{B}\right)^{s}-\left(\widehat{k}_{2} \odot \widehat{\boldsymbol{\omega}}_{B T}^{B}\right)^{s}-\widehat{\mathbf{f}}_{d}^{B}\right]+\left[\widehat{k} \odot\left(\widehat{\mathbf{q}}_{B T}-\widehat{1}\right)\right] \circ\left(\widehat{\mathbf{q}}_{B T} \widehat{\boldsymbol{\omega}}_{B T}^{B}\right) \\
& +\left(\widehat{\boldsymbol{c}} \odot \widehat{\mathbf{p}}_{B T}^{B}\right)^{s} \circ\left[\widehat{\mathbf{S}} \widehat{\boldsymbol{\omega}}_{B T}^{B}-\left(\widehat{k}_{1} \odot \widehat{\mathbf{p}}_{B T}^{B}\right)^{s}-\left(\widehat{k}_{2} \odot \widehat{\boldsymbol{\omega}}_{B T}^{B}\right)^{s}-\widehat{\mathbf{f}}_{d}^{B}\right]+\left(\widehat{\boldsymbol{c}} \odot \dot{\widehat{\mathbf{p}}}_{B T}^{B}\right)^{s} \circ\left(\mathbf{M}_{B} \widehat{\boldsymbol{\omega}}_{B T}^{B}\right) \\
= & -\left(\widehat{\boldsymbol{c}} \odot \widehat{\mathbf{p}}_{B T}^{B}\right) \circ\left(\widehat{k}_{1} \odot \widehat{\mathbf{p}}_{B T}^{B}\right)-\widehat{\boldsymbol{\omega}}_{B T}^{B} \circ\left(\widehat{k}_{2} \odot \widehat{\boldsymbol{\omega}}_{B T}^{B}\right)-\left(\widehat{\boldsymbol{\omega}}_{B T}^{B}+\widehat{\boldsymbol{c}} \odot \widehat{\mathbf{p}}_{B T}^{B}\right)^{s} \circ \widehat{\mathbf{f}}_{d}^{B}+Z,
\end{aligned}
$$

where $\quad Z=\left(\widehat{c} \odot \dot{\hat{\mathbf{p}}}_{B T}^{B}\right)^{s} \circ\left(\mathbf{M}_{B} \widehat{\boldsymbol{\omega}}_{B T}^{B}\right)+\left(\widehat{c} \odot \widehat{\mathbf{p}}_{B T}^{B}\right)^{s} \circ\left(\widehat{\mathbf{S}} \widehat{\boldsymbol{\omega}}_{B T}^{B}\right)$.

Based on the definition of $\widehat{\mathbf{S}}$ in (18) and $\mathbf{v}_{B T}^{B}=\dot{\mathbf{t}}_{B T}^{B}+\boldsymbol{\omega}_{B T}^{B} \times \mathbf{t}_{B T}^{B}$, there is

$$
\begin{gathered}
Z=\left(c_{v} \dot{\mathbf{t}}_{B D}^{B}+\varepsilon c_{\boldsymbol{\omega}} \dot{\mathbf{q}}_{B T}\right) \circ\left(m_{c} \mathbf{v}_{B D}^{B}+\varepsilon \mathbf{J}_{B} \boldsymbol{\omega}_{B T}^{B}\right)+\left(c_{v} \mathbf{t}_{B T}^{B}+\varepsilon c_{\boldsymbol{\omega}} \mathbf{q}_{B T}\right) \circ\left(\mathbf{S}_{1} \mathbf{v}_{B T}^{B}+\varepsilon \mathbf{S}_{2} \boldsymbol{\omega}_{B T}^{B}\right) \\
\leq \frac{3 c_{\boldsymbol{\omega}} \gamma_{J}}{2}\left\|\boldsymbol{\omega}_{B T}^{B}\right\|^{2}+3 c_{\boldsymbol{\omega}} \gamma_{J} \gamma_{\boldsymbol{\omega}}\left\|\mathbf{q}_{B T}\right\|\left\|\boldsymbol{\omega}_{B T}^{B}\right\|+c_{v} m_{B}\left\|\mathbf{v}_{B T}^{B}\right\|^{2}+2 c_{v} m_{B} \gamma_{\boldsymbol{\omega}}\left\|\mathbf{t}_{B T}^{B}\right\|\left\|\mathbf{v}_{B T}^{B}\right\| . \\
\dot{V} \leq-\|\mathbf{x}\|\left(\lambda_{\min }\left(\mathbf{A}^{\prime}\right)\|\mathbf{x}\|-\|\mathbf{B}\|\left\|\mathbf{x}_{d}\right\|\right) .
\end{gathered}
$$

Substituting (39) into (38) yields

$$
\dot{V}_{1} \leq-\mathbf{x}^{\mathrm{T}} \mathbf{A}^{\prime} \mathbf{x}+\mathbf{x}^{\mathrm{T}} \mathbf{B} \mathbf{x}_{d}
$$

where $\quad \mathbf{x}_{d}=\left[\begin{array}{c}\left\|\mathbf{f}_{d}^{B}\right\| \\ \left\|\boldsymbol{\tau}_{d}^{B}\right\|\end{array}\right], \mathbf{B}=\left[\begin{array}{cc}0 & c_{\boldsymbol{\omega}} \\ 0 & 1 \\ c_{v} & 0 \\ 1 & 0\end{array}\right]$ and

$$
\mathbf{A}^{\prime}=\left[\begin{array}{cccc}
c_{\omega} k_{q} & \left(\left(-3 c_{\omega} \gamma_{J} \gamma_{\omega}\right) / 2\right) & 0 & 0 \\
\left(\left(-3 c_{\omega} \gamma_{J} \gamma_{\omega}\right) / 2\right) & k_{\omega}-\left(\left(3 c_{\omega} \gamma_{J}\right) / 2\right) & 0 & 0 \\
0 & 0 & c_{v} k_{v} & -c_{v} m_{B} \gamma_{\omega} \\
0 & 0 & -c_{v} m_{B} \gamma_{\omega} & k_{v}-c_{v} m_{B}
\end{array}\right] \text {. }
$$

Similarly, it can be verified that the positives definiteness of $\mathbf{A}^{\prime}$ can be ensured by sufficiently small $c_{\boldsymbol{\omega}}$ and $c_{v}$. Denote the minimum eigenvalue of $\mathbf{A}^{\prime}$ as $\lambda_{\min }\left(\mathbf{A}^{\prime}\right)$; , then, there is
Based on (41) and (30), it can be obtained that

$$
\lim _{t \rightarrow \infty}\|\mathbf{x}\| \leq \frac{\|\mathbf{B}\|}{\lambda_{\min }\left(\mathbf{A}^{\prime}\right)} \lim _{t \longrightarrow \infty}\left\|f_{\sim}^{B}(t)\right\| \leq \frac{\|\mathbf{B}\|}{\lambda_{\min }\left(\mathbf{A}^{\prime}\right)} \frac{\left\|\dot{\overrightarrow{\mathbf{f}}}_{d}^{B}\right\|}{\lambda} .
$$

It can be seen from (42) that when $c_{\omega}$ and $c_{v}$ have been decided, the ultimate bound of $\mathbf{x}$ is entirely determined by the ultimate bound of $\mathbf{f}-d^{B}$. Therefore, the ultimate bound of $\mathbf{x}$ can be made smaller by properly increasing the value of $\lambda$.

Remark 4. To guarantee the positive definiteness of $\mathbf{A}$ in (37) and $\mathbf{A}^{\prime}$ in (40), the ranges of $c_{\boldsymbol{\omega}}$ and $c_{v}$ can be calculated as

$$
\begin{aligned}
& 0<c_{\boldsymbol{\omega}}<\min \left\{\frac{k_{\boldsymbol{\omega}}+\sqrt{k_{\omega}^{2}+2 \gamma_{J} k_{q}}}{\gamma_{J}}, \frac{4 k_{q} k_{\boldsymbol{\omega}}}{9 \gamma_{J}^{2} \gamma_{\boldsymbol{\omega}}^{2}+6 \gamma_{J} k_{q}}\right\}, \\
& 0<c_{v}<\min \left\{\frac{k_{v}+\sqrt{k_{v}^{2}+4 m_{B} k_{q}}}{2 m_{B}}, \frac{k_{r} k_{v}}{m_{B}^{2} \gamma_{\omega}^{2}+m_{B} k_{q}}\right\} .
\end{aligned}
$$


Note that in the proposed I\&I adaptive control scheme, since $c_{\omega}$ and $c_{v}$ are only used in the stability analysis rather than in the control law, they are not related to the control effect. From (43), it can be seen that the main constraints are the upper bounds and as long as $c_{\omega}$ and $c_{v}$ are close enough 0 , the positive definiteness of $\mathbf{A}$ and $\mathbf{A}^{\prime}$ can always be guaranteed. Therefore, the exact upper bounds of $c_{\boldsymbol{\omega}}$ and $c_{v}$ are actually not needed, which is also an advantage of the I\&I adaptive control scheme.

When the disturbances are constant, the second result of this paper is described in the following theorem.
Theorem 2. Consider the closed-loop system composed of relative motion models (14) and (16), disturbance estimator (32), and control law (33). Then, the tracking error states $\widehat{\mathbf{p}}_{B T}^{B} \longrightarrow \widehat{0}, \widehat{\boldsymbol{\omega}}_{B T}^{B} \longrightarrow \widehat{0}$ as $t \longrightarrow \infty$ for all initial conditions.

Proof. Consider the following candidate Lyapunov function:

$$
V_{2}=\frac{1}{2}\left(\widehat{\boldsymbol{\omega}}_{B T}^{B}\right)^{\mathrm{s}} \circ\left(\mathbf{M}_{B} \widehat{\boldsymbol{\omega}}_{B T}^{B}\right)+\left(\widehat{k}_{1} \odot\left(\widehat{\mathrm{q}}_{B T}-\widehat{1}_{H}\right)\right) \circ\left(\widehat{\mathrm{q}}_{B T}-\widehat{1}_{H}\right) .
$$

The time derivative of $V_{2}$ can be calculated as

$$
\begin{aligned}
\dot{V}_{2} & =-\left(\hat{\boldsymbol{\omega}}_{B T}^{B}\right)^{s} \circ\left(\left(\hat{k}_{2} \odot \hat{\boldsymbol{\omega}}_{B T}^{B}\right)^{s}+\underline{\mathbf{f}}_{d}^{B}\right) \leq-\underline{k}_{2}\left\|\hat{\boldsymbol{\omega}}_{B T}^{B}\right\|^{2}+\left\|\hat{\boldsymbol{\omega}}_{B T}^{B}\right\|\left\|\hat{\mathbf{f}}_{d}^{B}\right\|, \\
& \leq-\left(-\underline{k}_{2}-\chi\right)\left\|\hat{\boldsymbol{\omega}}_{B T}^{B}\right\|^{2}+\frac{\left\|\widehat{\mathbf{f}}_{d}^{B}\right\|^{2}}{4 \chi} \leq \frac{\left\|\hat{\mathbf{f}}_{d}^{B}\right\|^{2}}{4 \chi},
\end{aligned}
$$

where Young's inequality has been applied, $\underline{k}_{2}=\min \left\{k_{\omega}, k_{v}\right\}$, and $\chi \in\left(0, \underline{k}_{2}\right)$. Integrating both sides of $\dot{V}_{2} \leq\left(\left\|\widehat{\mathbf{f}}_{d}^{B}\right\|^{2} / 4 \chi\right)$ on $[0, t]$ leads to

$$
V_{2}(t)-V_{2}(0) \leq \int_{0}^{t} \frac{\left\|\hat{\mathbf{f}}_{d}^{B}\right\|^{2}}{4 \chi} \mathrm{d} \tau
$$

When the disturbances are constant, according to (31), $\left\|\widehat{\mathbf{f}}_{d}^{B}\right\|^{2}$ is exponentially convergent; therefore, the right hand of $(46)$ is integrable on $[0, \infty]$. As a result, $V_{2}(t)$ is bounded on $[0, \infty]$. Hence, the system states $\widehat{\mathbf{q}}_{B T}$ and $\widehat{\boldsymbol{\omega}}_{B T}^{B}$ are also bounded.

On the other hand, when $\widehat{\mathbf{f}}_{d}^{B}=\widehat{0}$, for same candidate Lyapunov function $V_{2}$ in (44), there is $\dot{V}_{2}=-\left(\widehat{\boldsymbol{\omega}}_{B T}^{B}\right)^{s} \circ\left(\widehat{k}_{2} \odot \widehat{\boldsymbol{\omega}}_{B T}^{B}\right)^{s} \leq 0$. Therefore, $V_{2}, \widehat{\boldsymbol{\omega}}_{B T}^{B}, \widehat{\mathbf{q}}_{B T}, \dot{\boldsymbol{\mathbf { q }}}_{B T}$, and $\dot{V}_{2}$ are all bounded. Since $\dot{\hat{\boldsymbol{\omega}}}_{T I}^{T}$ is bounded, it follows from (16) that $\dot{\hat{\boldsymbol{\omega}}}_{B T}^{B}$ is bounded. Therefore, $\ddot{V}_{2}$ is bounded. Then, it follows from Barbalat's lemma that $\lim _{t \rightarrow \infty} \dot{V}_{2}=0$, and thus $\lim _{t \longrightarrow \infty} \widehat{\boldsymbol{\omega}}_{B T}^{B}=\widehat{0}$. Furthermore, since $\ddot{\widehat{\boldsymbol{\omega}}}_{B T}^{B}$ is also bounded, by Barbalat's lemma, there is $\lim _{t \rightarrow \infty} \dot{\widehat{\boldsymbol{\omega}}}_{B T}^{B}=\widehat{0}$, and thus $\lim _{t \rightarrow \infty} \widehat{\mathbf{p}}_{B T}^{B}=\widehat{0}$ according to (16).

In conclusion, when $\lim _{t \rightarrow \infty} \widehat{\mathbf{f}}_{d}^{B}=\widehat{0}$, all system states are bounded. When ${\underset{\sim}{\sim}}_{d}^{B}=\widehat{0}, \quad \lim _{t \rightarrow \infty}\left(\widehat{\mathbf{p}}_{B T}^{B}, \widehat{\boldsymbol{\omega}}_{B T}^{B}\right)=(\widehat{0}, \widehat{0})$. Therefore, according to the I\&I adaptive stability theorem [39], when $\lim _{t \longrightarrow \infty} \widehat{\mathbf{f}} d^{B}=\widehat{0}$, there is $\lim _{t \rightarrow \infty}\left(\widehat{\mathbf{p}}_{B T}^{B}, \widehat{\boldsymbol{\omega}}_{B T}^{B}\right)=(\widehat{0}, \widehat{0})$.

\section{Simulation Results}

The effectiveness and features of the proposed I\&I adaptive pose tracking control scheme composed of (32) and (33) are verified by numerical simulations and comparisons with two existing dual quaternion-based controllers. In the case of constant disturbances, the proposed control law is compared with a CEbased adaptive controller designed in [28]. In the case of timevarying disturbances, it is compared with a SMC given in [32].

The objective is to track the pose of a target spacecraft, which is orbiting in a highly eccentric Molniya orbit, and the orbit elements are given in Table 1. The target body fixed frame is assumed to be aligned with its orbit frame. The mass and inertial matrix of the chaser spacecraft are given as $m_{B}=100 \mathrm{~kg}, \mathbf{J}_{B}=\left[\begin{array}{ccc}22 & 0.2 & 0.5 \\ 0.2 & 20 & 0.4 \\ 0.5 & 0.4 & 23\end{array}\right] \mathrm{kg} \cdot \mathrm{m}^{2}$.

The initial conditions of the relative motion are set as $\mathbf{q}_{B T}(0)=\left[\begin{array}{llll}0.3153 & 0.2829 & -0.5659 & 0.7073\end{array}\right]^{\mathrm{T}}, \quad \mathbf{r}_{B T}^{B}(0)=$ $\left[\begin{array}{lll}-12 & 8 & -5\end{array}\right]^{\mathrm{T}} \mathrm{m}, \boldsymbol{\omega}_{B T}^{B}(0)=\left[\begin{array}{lll}0.01 & -0.03 & 0.02\end{array}\right](\mathrm{rad} / \mathrm{s})$, and $\mathbf{v}_{B T}^{B}(0)=\left[\begin{array}{lll}0.4 & -0.2 & 0.1\end{array}\right](\mathrm{m} / \mathrm{s})$. The gains for the pose tracking controller (33) are selected as $k_{q}=1, k_{r}=2.8$, $k_{\omega}=6$, and $k_{v}=20$.

In order to evaluate the control effort, the energy consumption is defined as

$$
E(t)=\int_{0}^{t}\left[\left(\widehat{\theta} \odot \widehat{\mathbf{f}}_{u}^{B}(s)\right) \circ\left(\widehat{\theta} \odot \widehat{\mathbf{f}}_{u}^{B}(s)\right)\right] \mathrm{d} s,
$$

where $\widehat{\theta}=1(\mathrm{~m})+\varepsilon 1$ is used to unify the units of the energy to $\mathrm{N}^{2} \mathrm{~m}^{2} \mathrm{~s}$.

5.1. Simulations in the Case of Constant Disturbance. The adaptive disturbance estimation law designed based on certainty equivalence principle is given as [28]

$$
\widehat{\mathbf{f}}_{d}^{B}=\widehat{\alpha} \odot\left(\widehat{\boldsymbol{\omega}}_{B T}^{B}+\widehat{c} \odot \mathbf{p}_{B T}^{B}\right)^{\mathcal{s}},
$$

where $\widehat{\alpha}=\alpha_{f}+\varepsilon \alpha_{\tau}, \alpha_{f}$ and $\alpha_{\tau}$ are positive adaptive gains, and $\widehat{c}=c_{\omega}+\varepsilon 2 c_{v}$ is the same as that defined in (35). The CE- 
based adaptive controller for comparison is composed of pose tracking law (33) and the disturbance estimation law (48).

As stated in Remark 4, in the proposed I\&I adaptive control scheme, $\widehat{c}$ is only used for stability analysis; therefore, the positive definiteness of $\mathbf{A}$ and $\mathbf{A}^{\prime}$ can always be ensured by small enough $c_{\boldsymbol{\omega}}$ and $c_{v}$ without knowing their exactly upper bounds. However, in the CE-based control law, $\widehat{c}$ is also involved in the adaption law as shown in (48), whose value will also influence the estimation effect. Thus, for the CE-based control law, the upper bounds of $c_{\omega}$ and $c_{v}$ have to be specified, which means the value of $\gamma_{\omega}$ needs to be known in advance.

According to the target orbit elements, there is $\gamma_{\omega}=0.0004$. Then, based on (43), the ranges of $c_{\omega}$ and $c_{v}$ can be calculated as $0<c_{\omega}<0.172$ and $0<c_{v}<0.295$. The parameters for the CE controller are set as $\widehat{c}=0.17+\varepsilon 0.29$ and $\alpha=0.28+\varepsilon 0.4$.

Note that to guarantee the positive definiteness of $\mathbf{A}$ in (37) and $\mathbf{A}^{\prime}$ in (40), $c_{\boldsymbol{\omega}}$ and $c_{v}$ have to be small enough. In the proposed I\&I adaptive control scheme, $\widehat{c}$ is only used for stability analysis; therefore, the positive definiteness of $\mathbf{A}$ and $\mathbf{A}^{\prime}$ can always be ensured by small enough $c_{\boldsymbol{\omega}}$ and $c_{v}$ without knowing the exactly value of $\gamma_{\omega}$. However, in the CE-based control law, $\widehat{c}$ is also involved in the adaption law as shown in (48), whose value will also influence the estimation effect. Thus, for the CE-based control law, the value ranges of $c_{\omega}$ and $c_{v}$ have to specified, which needs the value of $\gamma_{\omega}$ in advance.

According to the target orbit elements, there is $\gamma_{\omega}=0.0004$. Then, based on (43), the ranges of $c_{\omega}$ and $c_{v}$ can be calculated as $0<c_{\omega}<0.172$ and $0<c_{v}<0.295$. The parameters for the CE controller are set as $\widehat{c}=0.17+\epsilon 0.29$ and $\alpha=0.28+\epsilon 0.4$.

The constant disturbances exerted on the chaser spacecraft are set as $\mathbf{f}_{d}^{B}=\left[\begin{array}{lll}0.4 & 0.5 & -0.6\end{array}\right]^{\mathrm{T}} \mathrm{N}$ and $\tau_{d}^{B}=\left[\begin{array}{lll}-0.06 & 0.05 & 0.08\end{array}\right]^{\mathrm{T}} \mathrm{Nm}$. For both adaption laws, the initial value of the disturbance estimation is set to $\underline{\hat{\mathbf{f}}}_{d}^{B}(0)=\widehat{0}$.

Figure 1 shows the global result of the tracking trajectory. Figures 2-7 present the time responses of relative attitude and position, relative angular velocity and linear velocity, and control force and torque under the two adaptive controllers which are composed of the same pose tracking control law and different disturbance estimation laws. Although both controllers achieve the tracking control objective, the proposed controller has smoother transient responses, shorter settling time, and higher tracking precision. After $120 \mathrm{~s}$, the errors of relative attitude and position of the proposed controller are smaller than $5 \times 10^{-7}$ and $1.5 \times 10^{-4} \mathrm{~m}$ and those of the CE-based controller are $1.5 \times$ $10^{-4}$ and $4 \times 10^{-3} \mathrm{~m}$. The energy costs of the proposed and the CE-based controllers are $29.6 \mathrm{~N}^{2} \mathrm{~m}^{2} \mathrm{~s}$ and $34.1 \mathrm{~N}^{2} \mathrm{~m}^{2} \mathrm{~s}$, and the former is $86.8 \%$ of the latter. Such significant performance improvements without increasing the control efforts are due to the fact that I\&I-based adaption law can estimate the disturbances quickly, smoothly, and accurately. As shown in Figures 8 and 9, disturbance estimation errors under the I\&I-based adaption law converge to zero at about $30 \mathrm{~s}$ in exponential form while those under the CE-based adaption law converge after $100 \mathrm{~s}$ with large overshoots and oscillatory transient responses, which lead to the deterioration of the relative motion states and more energy consumption.

By comparison of the I\&I-based and CE-based adaption laws (32) and (48), it can be observed that the I\&I-based one is driven by the estimation errors rather than the system states like the CE-based one. This brings the I\&I-based adaptive estimator another nice property, that is, the socalled "parameter lock," which means when the true values are reached, the parameter estimations automatically stop updating and get locked on their corresponding true values. This feature of the I\&I adaption law is clearly shown in Figure 10 where the initial disturbance estimations of both estimators are set as the true values. It can be seen that the CE-based adaption law still drives the estimation away from the true value while the I\&I one can keep the estimation staying at the true values.

5.2. Simulations in the Case of Time-Varying Disturbance. The SMC for comparison is given as [32]

$$
\left\{\begin{array}{l}
\hat{f}_{u}^{B}=-\left(\widehat{k}_{k} \odot \widehat{s}\right)^{s}-\left(\widehat{k}_{\sigma} \odot \operatorname{sgm}(\widehat{s})\right)^{s}+\widehat{\boldsymbol{\omega}}_{B I}^{B} \times \widehat{\mathbf{M}}_{B} \widehat{\boldsymbol{\omega}}_{B I}^{B}+\widehat{\mathbf{M}}_{B}\left(\widehat{\mathbf{C}}_{B T} \dot{\boldsymbol{\mathbf { \omega }}}_{T I}^{T}-\widehat{\boldsymbol{\omega}}_{B T}^{B} \times \widehat{\boldsymbol{\omega}}_{T I}^{B}\right)-\widehat{\mathbf{M}}_{B} \dot{\overline{\mathbf{p}}}_{B T}^{B}-\widehat{\mathbf{M}}_{B} \hat{\mathbf{a}}_{g}^{B}-3 \mu \frac{\hat{\mathbf{t}}_{B I}^{B} \times \mathbf{M}}{\| \hat{\mathbf{t}}_{B I}^{B}} \hat{\mathbf{t}}_{B I}^{B} \|^{5}-\widehat{\mathbf{M}}_{B} \hat{\mathbf{a}}_{J_{2}}^{B}, \\
\widehat{\boldsymbol{s}}=\widehat{\boldsymbol{\omega}}_{B T}^{B}+\widehat{k}_{a} \odot \widehat{\mathbf{p}}_{B T}^{B},
\end{array}\right.
$$

where $\widehat{k}_{\star}=k_{\star}+\varepsilon k_{\star}^{\prime}, \star \in\{k, \sigma, a\}$, and $k_{\star}$ and $k_{\star}^{\prime}$ are positive constants. $\operatorname{sgm}(\widehat{\mathcal{s}})=\left[\operatorname{sgm}\left(s_{1}\right), \operatorname{sgm}\left(s_{2}\right), \ldots, \operatorname{sgm}\right.$ $\left.\left(s_{6}\right)\right]^{\mathrm{T}}$, and $\operatorname{sgm}(x)=(x /(|x|+\Delta))$ with $\Delta$ being a small positive constant is the sigmoid function used for replacing the sign function and alleviating the chattering problem.
With this modification, the tracking errors can only converge to a neighbourhood of the origin.

Note that to effectively reject the disturbance, $\widehat{k}_{\sigma}$ has to satisfy

$$
k_{\sigma}>D_{\tau}, k_{\sigma}^{\prime}>D_{f},
$$


TABLE 1: Orbit elements of the target spacecraft.

\begin{tabular}{lr}
\hline Orbit elements & Values \\
\hline Semimajor axis $(\mathrm{km})$ & 26553.4 \\
Eccentricity & 0.73 \\
Inclination & 63.4 \\
Argument of perigee (deg) & 270 \\
RAAN (deg) & 329.6 \\
True anomaly (deg) & 100.5 \\
\hline
\end{tabular}

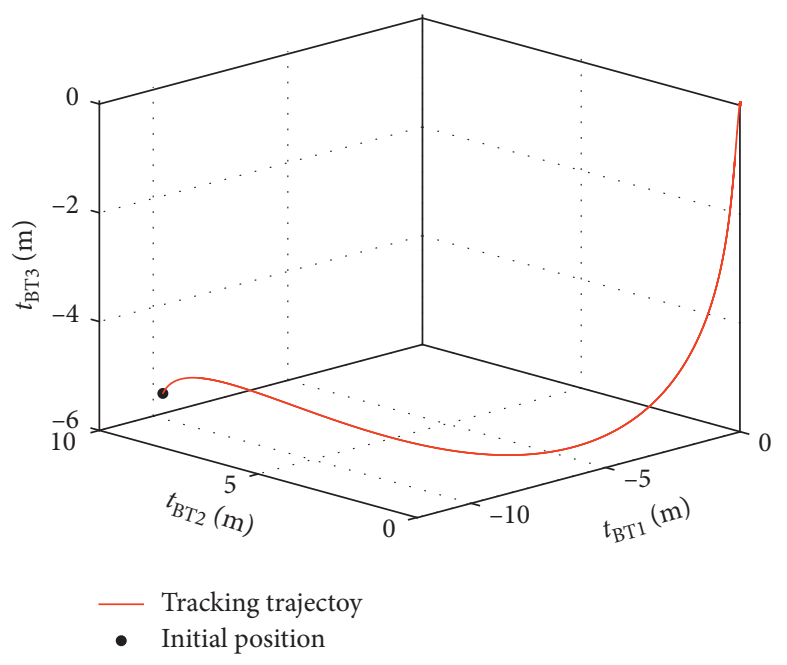

(a)

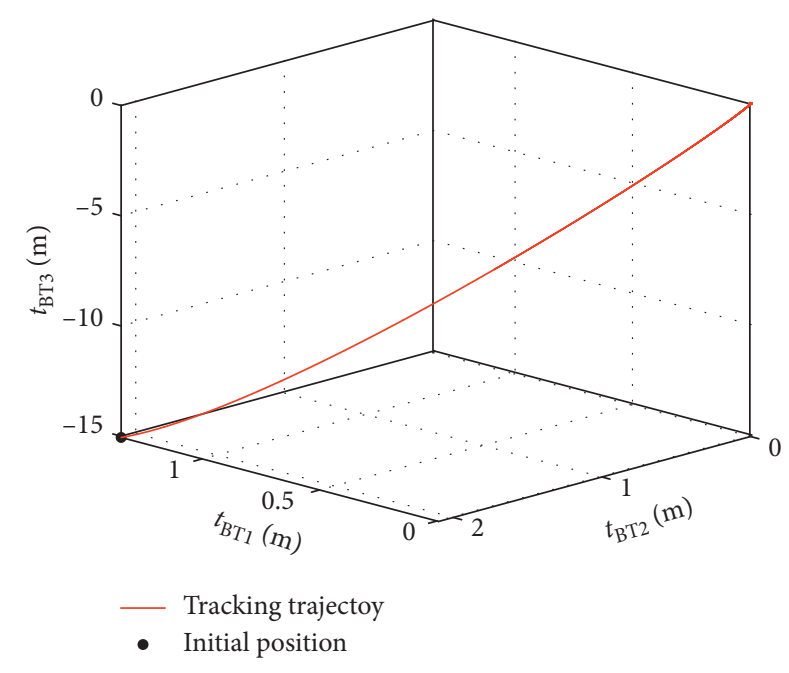

(b)

Figure 1: Global result of the tracking trajectory. (a) Observed in the chaser body fixed frame. (b) Observed in the target body fixed frame.

where $D_{\tau}$ and $D_{f}$ are the upper bounds of disturbance force and torque, which have to be known in advance for the design of the SMC (49). The time-varying disturbances are set as

$$
\begin{aligned}
& \mathbf{f}_{d}^{B}(t)=\left[\begin{array}{c}
0.4 \sin \left(0.03 t+\frac{\pi}{3}\right)+0.4 \\
-0.5 \sin (0.02 t+\pi)+0.3 \\
0.7 \sin \left(0.04 t+\frac{4 \pi}{3}\right)-0.2
\end{array}\right] \mathrm{N}, \\
& \boldsymbol{\tau}_{d}^{B}(t)=\left[\begin{array}{c}
0.05 \sin \left(0.02 t+\frac{\pi}{6}\right)+0.03 \\
0.06 \sin \left(0.03 t+\frac{\pi}{2}\right)+0.02 \\
-0.04 \sin (0.05 t+\pi)-0.05
\end{array}\right] \mathrm{Nm} .
\end{aligned}
$$

The upper bounds of disturbances are set as $D_{f}=1$ and $D_{\tau}=0.1$. The other parameters for the SMC are set as $\widehat{k}_{a}^{\tau}=0.3+\varepsilon 0.4 \widehat{k}_{k}=3+\varepsilon 8 k_{a}=0.1+\varepsilon 1$, and $\Delta=0.003 . \Delta$ is set as the minimum value which can eliminate the chattering. And the control gains of the SMC are tuned such that its settling time is approximately identical with that of the proposed controller.

The time histories of relative motion states under two controllers are presented in Figures 11 and 12. The steady state errors of relative attitude and position and relative angular and linear velocities of proposed controller are around $1 \times 10^{-4}, 5 \times 10^{-4} \mathrm{~m}, 5 \times 10^{-6} \mathrm{rad} / \mathrm{s}$, and $4 \times 10^{-5}$ $\mathrm{m} / \mathrm{s}$ and those of the SMC are around $4 \times 10^{-3}, 0.02 \mathrm{~m}, 3 \times$ $10^{-4} \mathrm{rad} / \mathrm{s}$, and $1 \times 10^{-3} \mathrm{~m} / \mathrm{s}$, which are much larger. It can be seen that in the presence of quite large time-varying disturbances, the SMC has to sacrifice the control precision for chattering alleviation while the proposed I\&I adaptive controller can still achieve relatively high control precision. The control forces and torques under the two controllers are shown in Figures 13 and 14. The proposed controller generates smaller and smoother control forces and torques than the SMC. Energy consumptions of the proposed controller and SMC for a total simulation time of $400 \mathrm{~s}$ are $26.4 \mathrm{~N}^{2} \mathrm{~m}^{2} s$ and $36.6 \mathrm{~N}^{2} \mathrm{~m}^{2} s$, respectively. The energy consumption of the proposed controller is $72.1 \%$ of that of the SMC.

Besides, Figure 15 compares the converge process of I\&I-based disturbance estimation errors with different $\lambda$ and verifies that the final estimation errors for the timevarying disturbances can be made smaller by choosing a larger $\lambda$. 


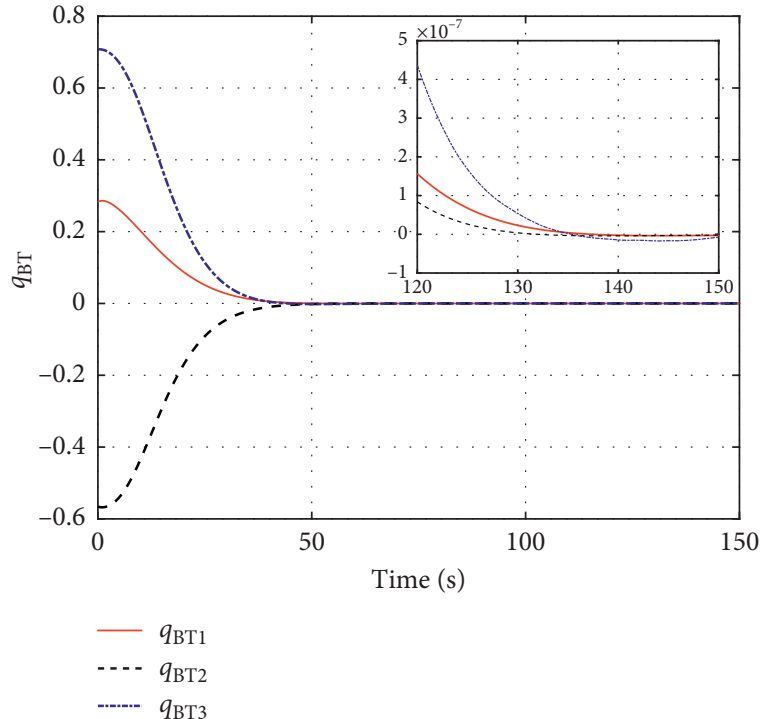

(a)

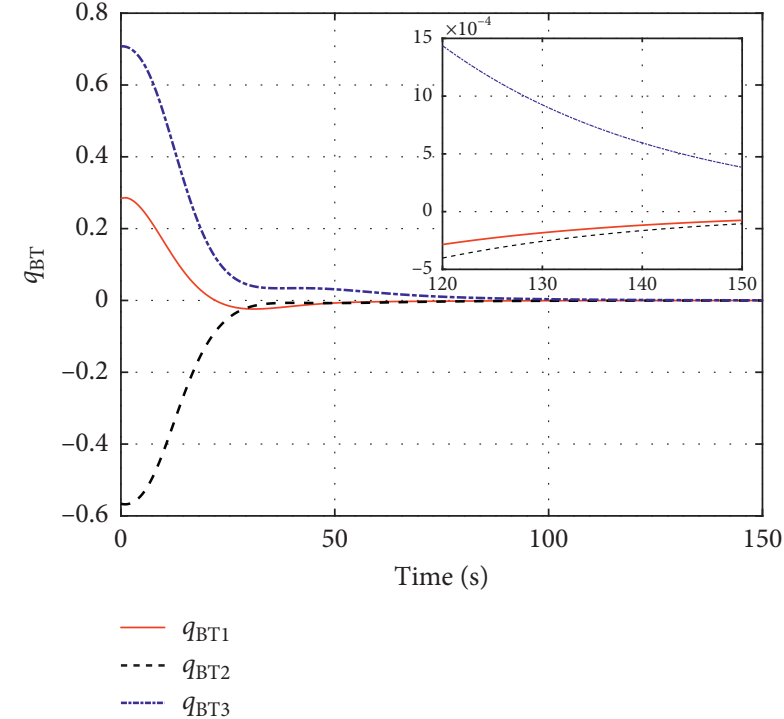

(b)

FIgUre 2: Relative attitude. (a) Proposed I\&I adaptive controller. (b) CE-based adaptive controller.

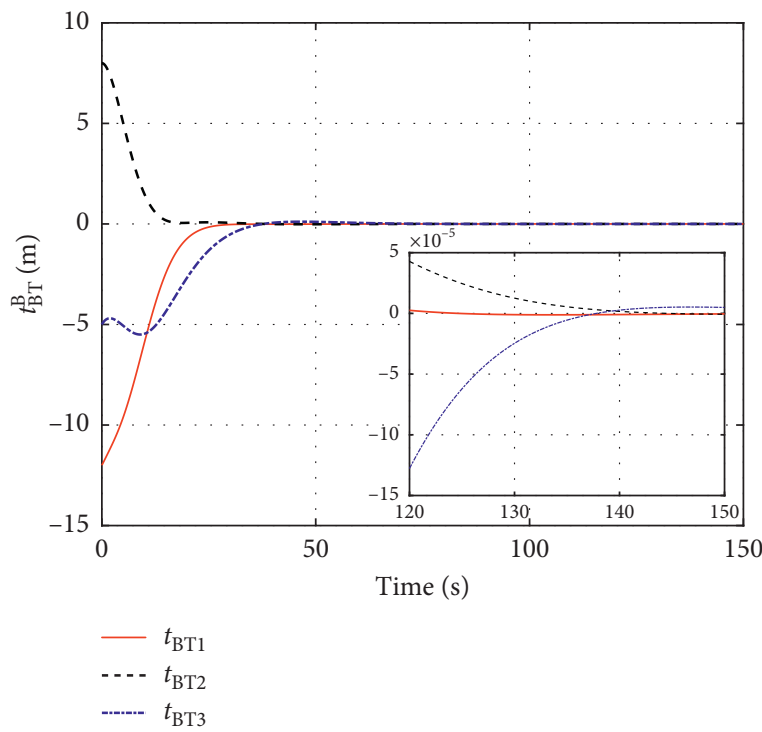

(a)

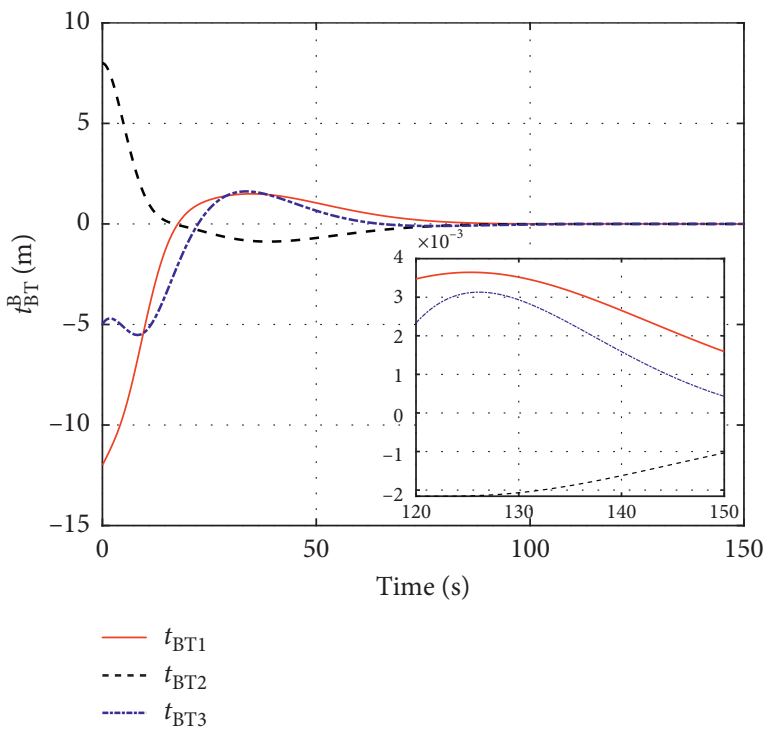

(b)

FIgURE 3: Relative position. (a) Proposed I\&I adaptive controller. (b) CE-based adaptive controller. 


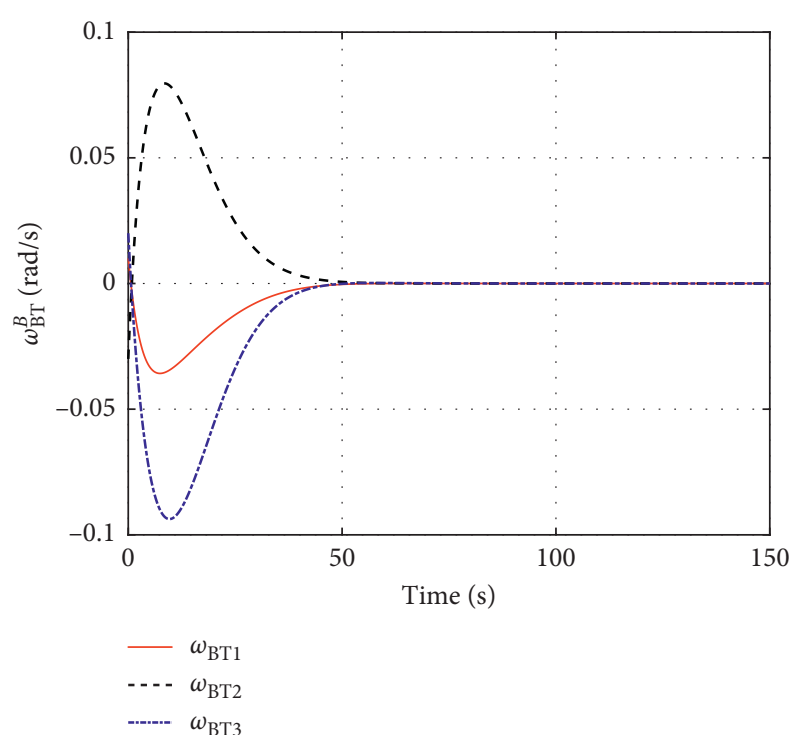

(a)

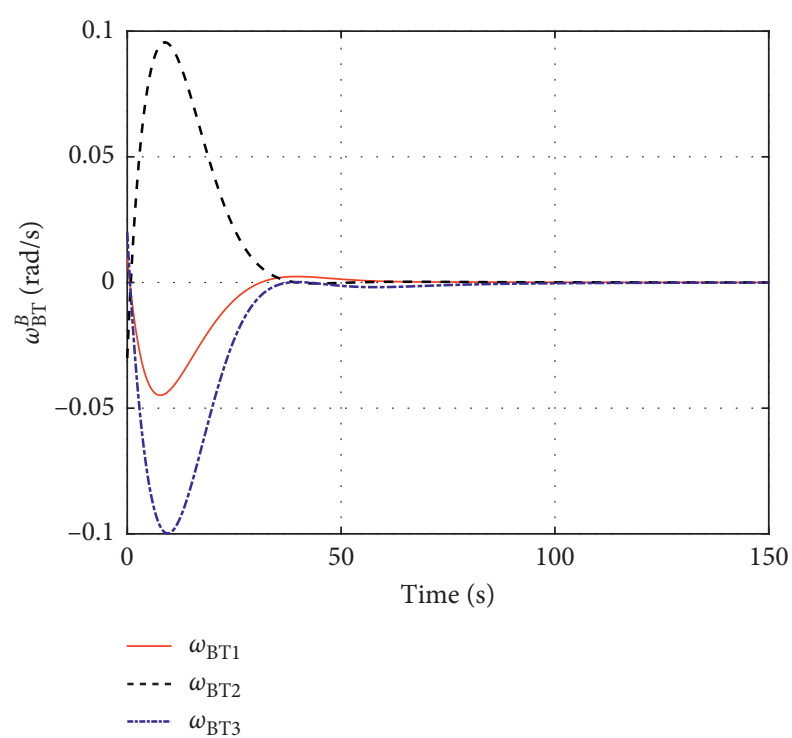

(b)

FIGURE 4: Relative angular velocity. (a) Proposed I\&I adaptive controller. (b) CE-based adaptive controller.
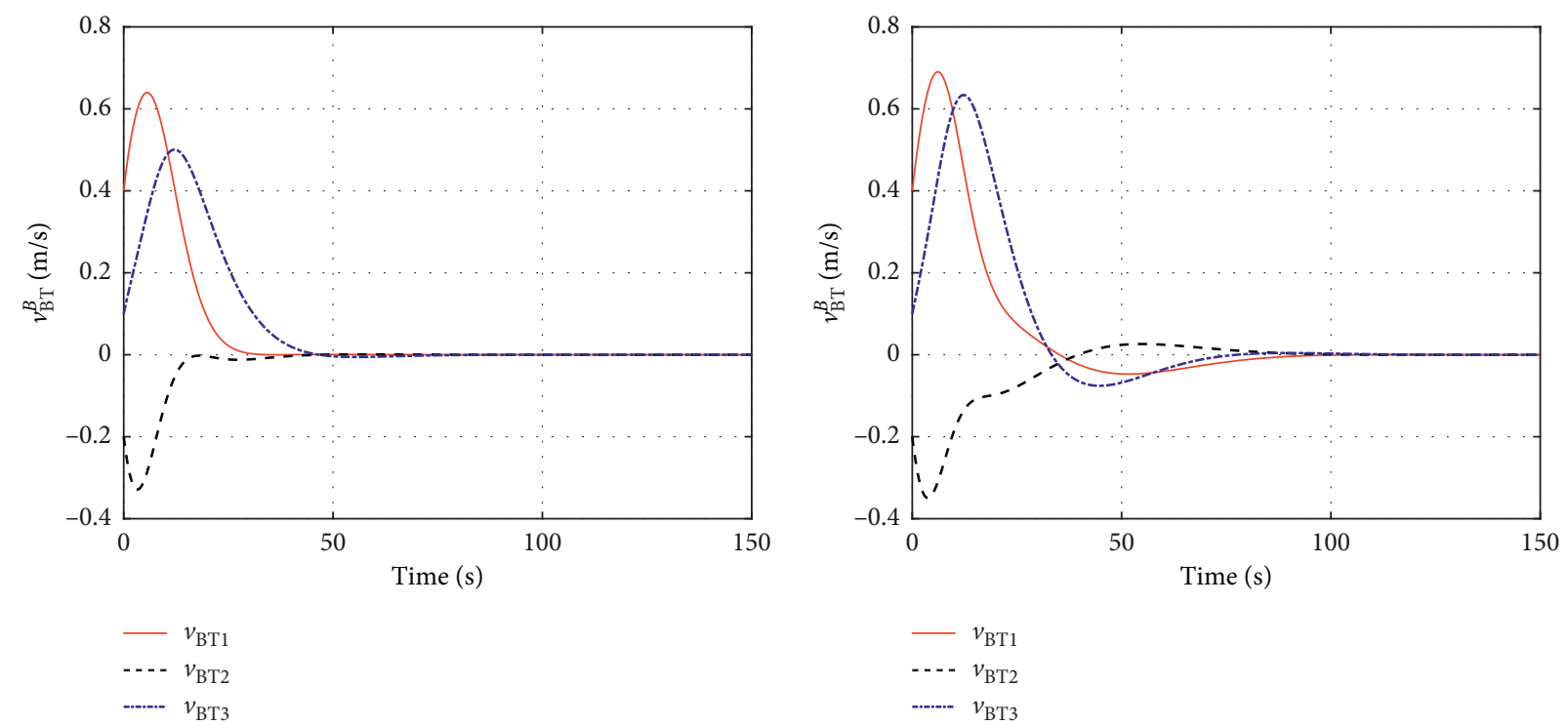

(a)

(b)

Figure 5: Relative linear velocity. (a) Proposed I\&I adaptive controller. (b) CE-based adaptive controller. 


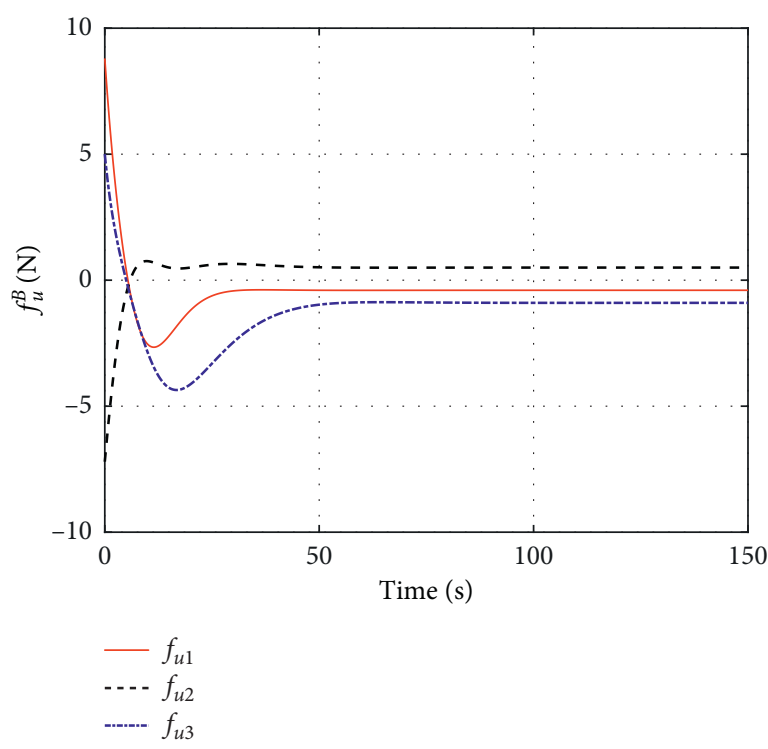

(a)

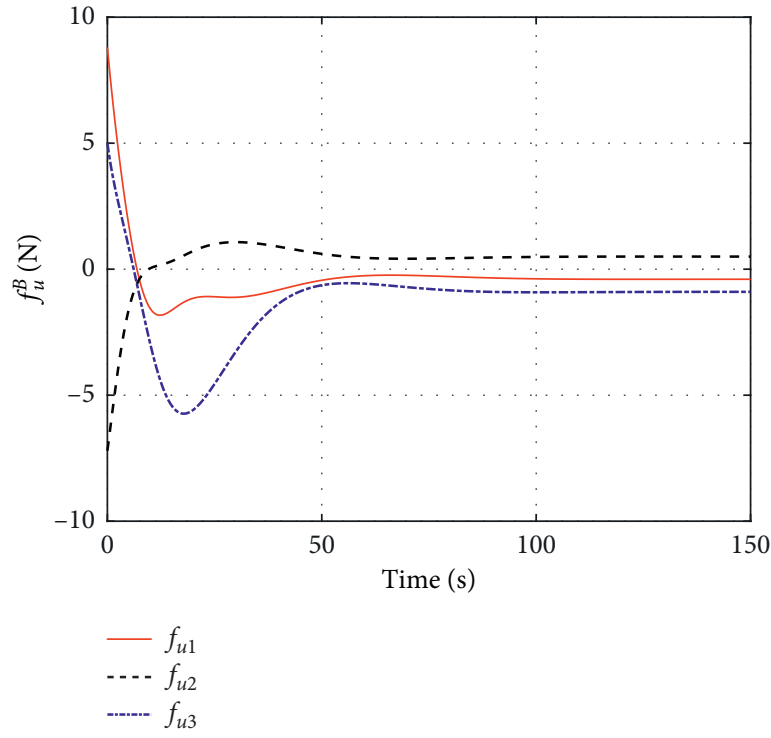

(b)

Figure 6: Control force. (a) Proposed I\&I adaptive controller. (b) CE-based adaptive controller.

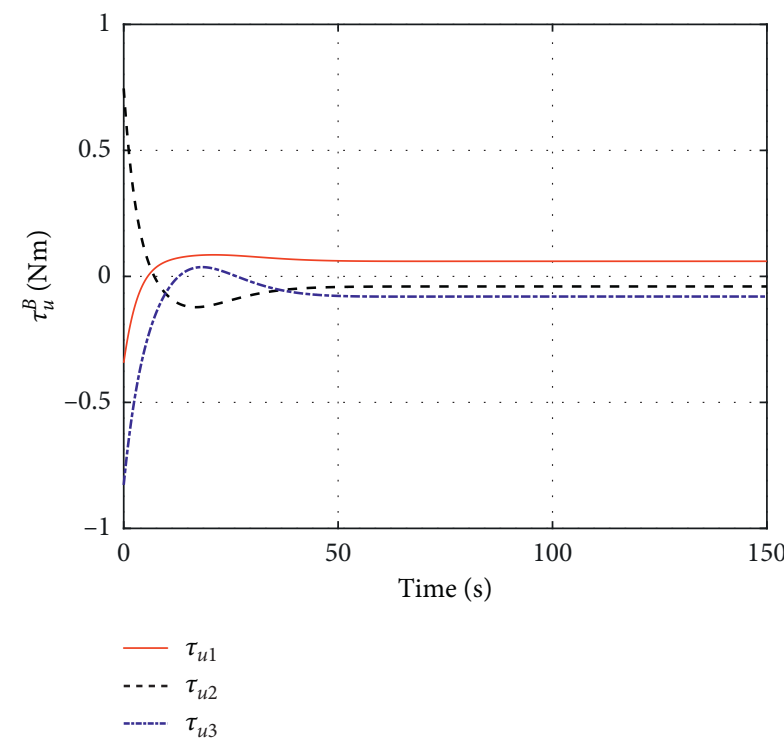

(a)

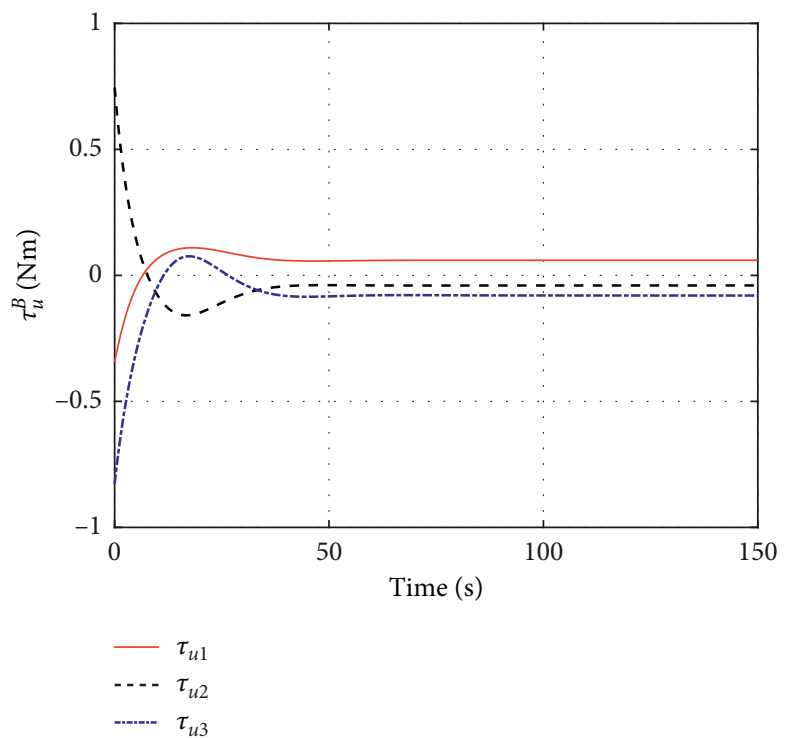

(b)

Figure 7: Control torque. (a) Proposed I\&I adaptive controller. (b) CE-based adaptive controller. 

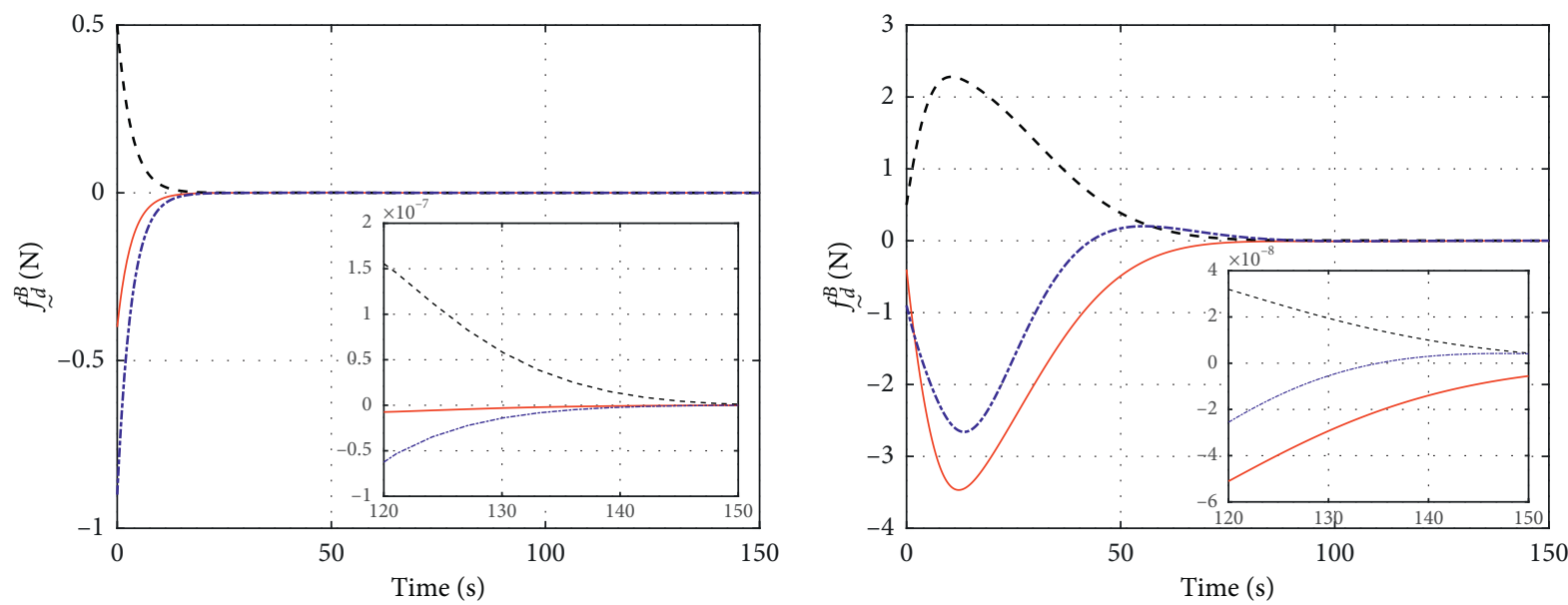
$-f_{d 1}$
$-f_{d 1}$
$\cdots f_{d 2}$
-... $f_{d 2}$
$\ldots-f_{d 3}$
$\cdots f_{d 3}$

(a)

(b)

Figure 8: Disturbance force estimation errors. (a) I\&I-based adaption law. (b) CE-based adaption law.

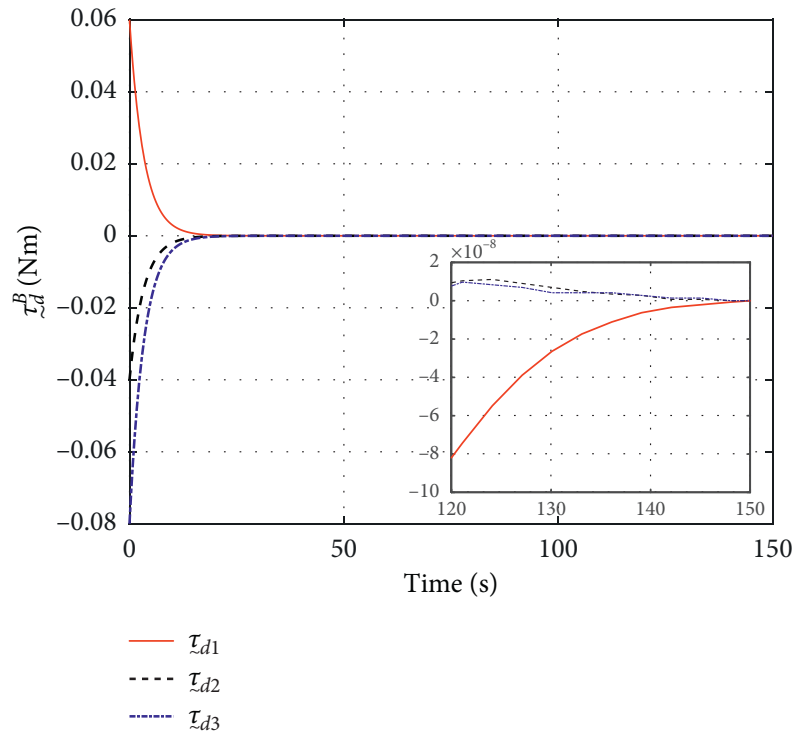

(a)

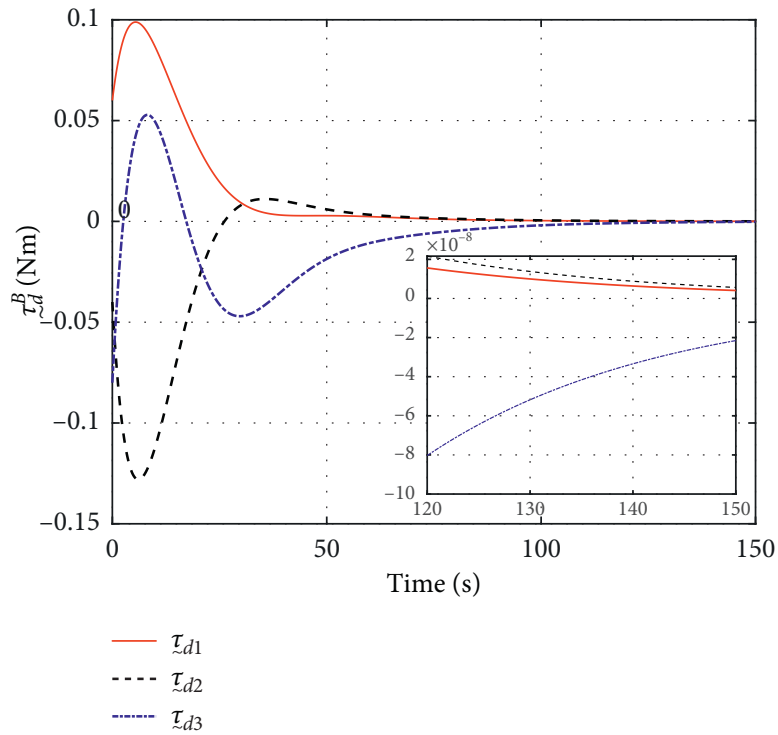

(b)

FIgURE 9: Disturbance torque estimation errors. (a) I\&I-based adaption law. (b) CE-based adaption law. 


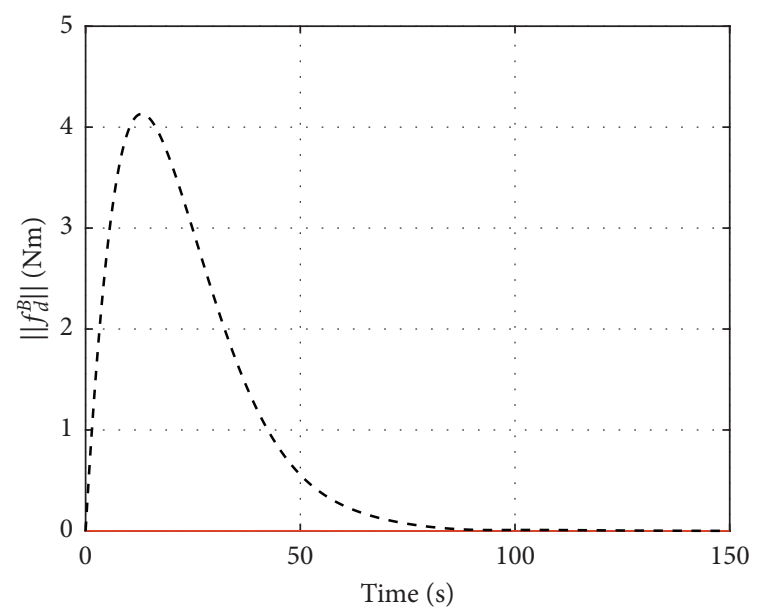

I\&I

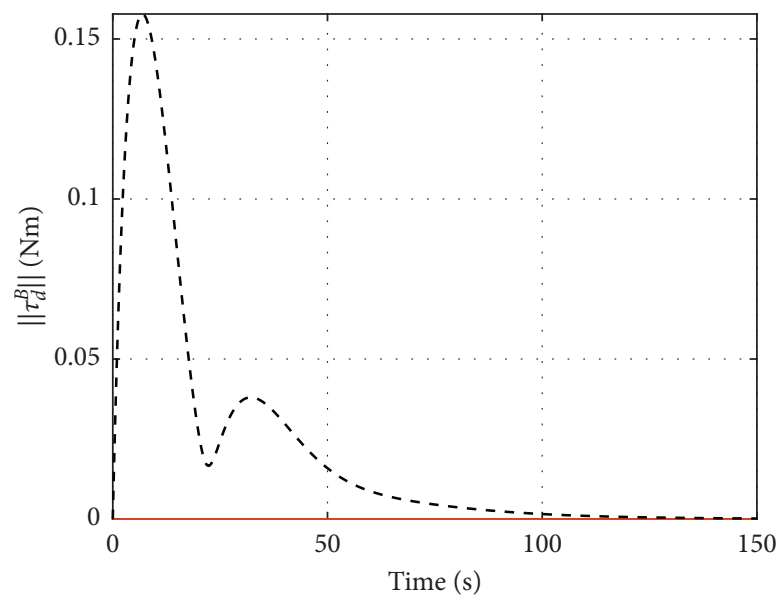

- I\&I

(a)

(b)

FIGURE 10: Disturbance estimation error norms under two adaption laws when $\widehat{f}_{-}^{B}(0)=\hat{f}_{d}^{B}$.

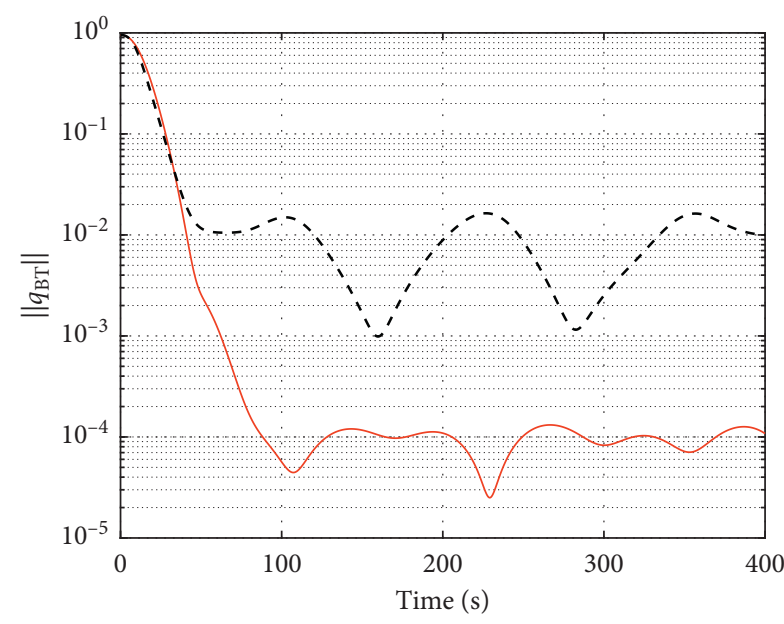

- I\&I

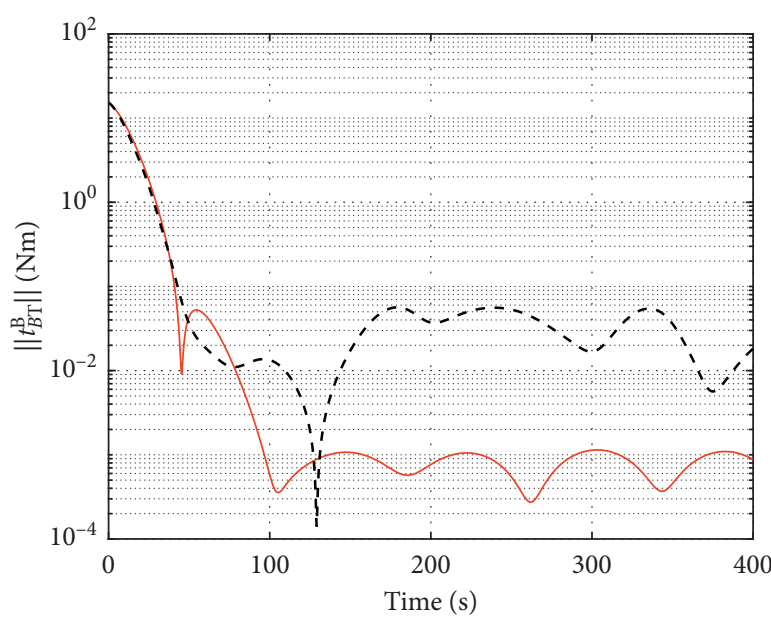

- I\&I

(a)

(b)

FIGURE 11: Relative pose norms under different controllers. 


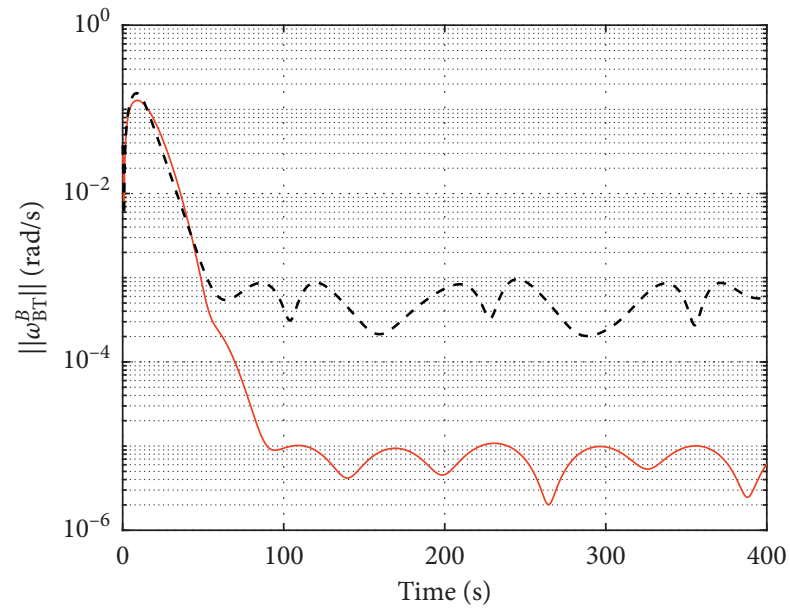

- I\&I

.... SMC

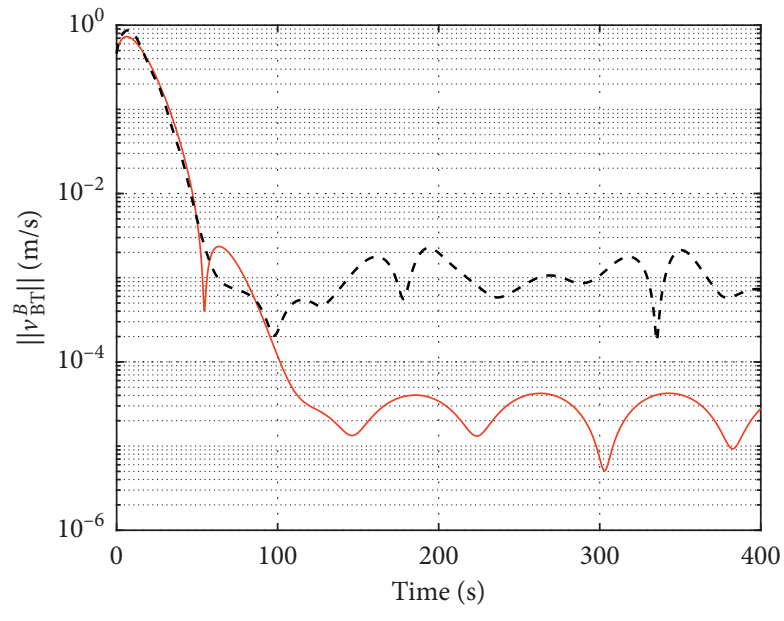

— I\&I

(a)

(b)

FIGURE 12: Relative dual velocity norms under different controllers.

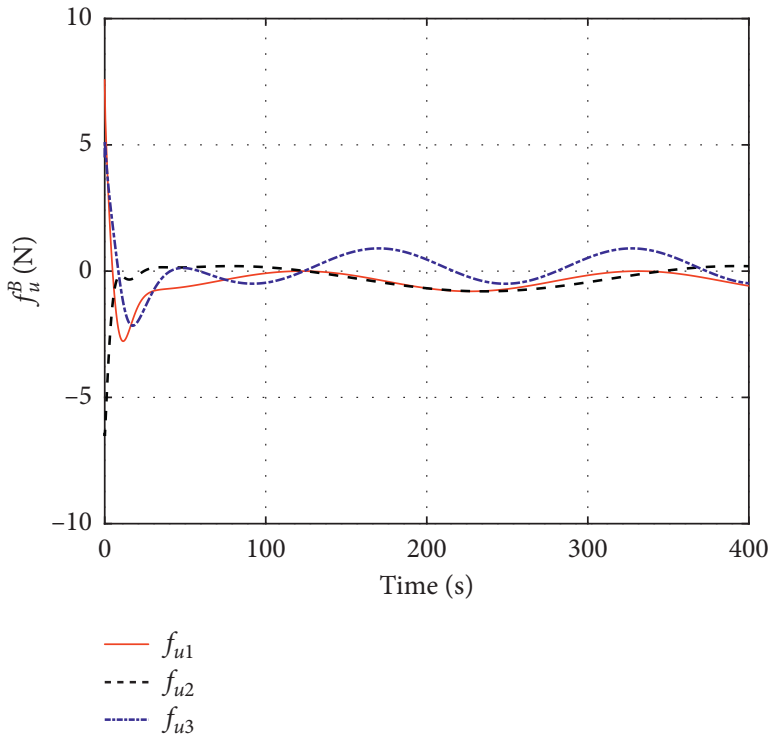

(a)

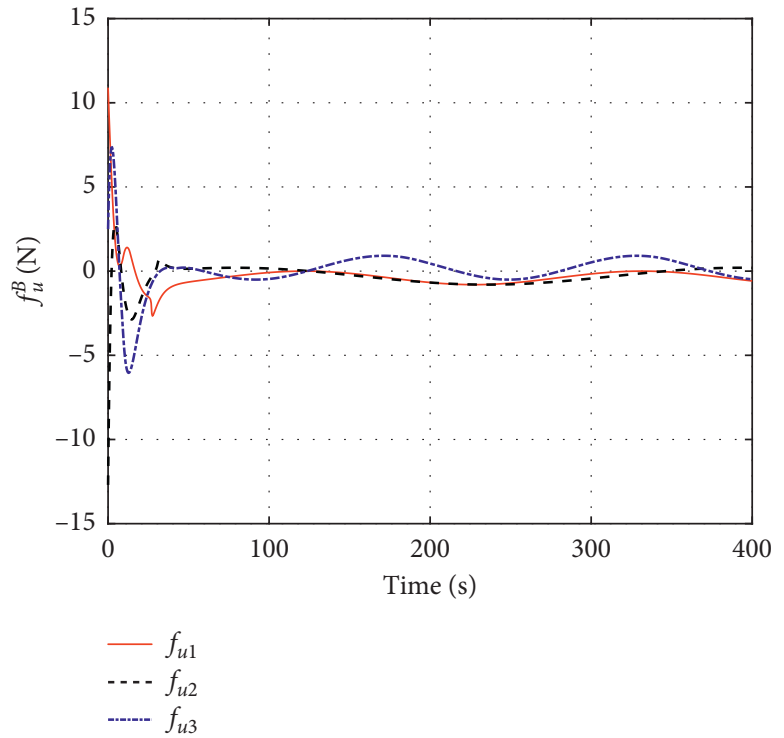

(b)

Figure 13: Control force. (a) Proposed I\&I adaptive controller. (b) SMC. 


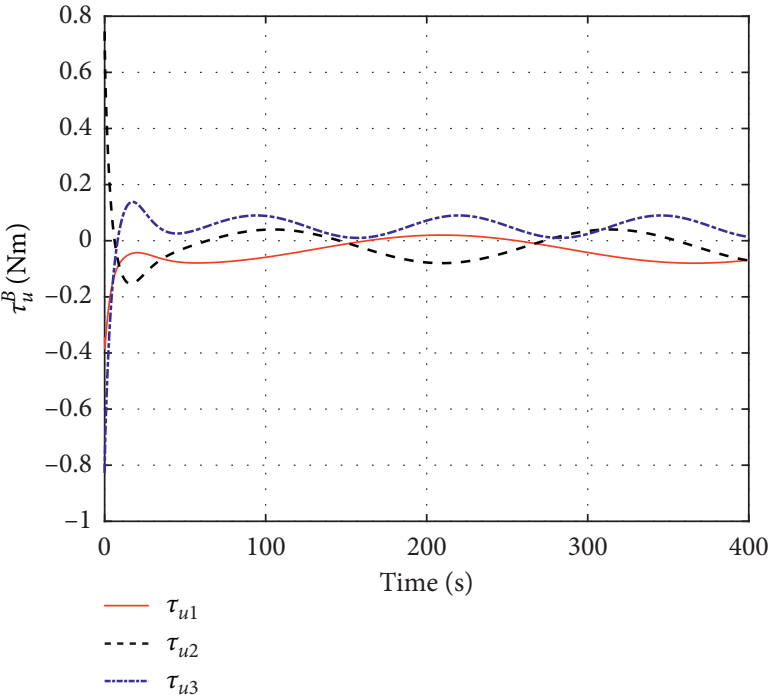

(a)

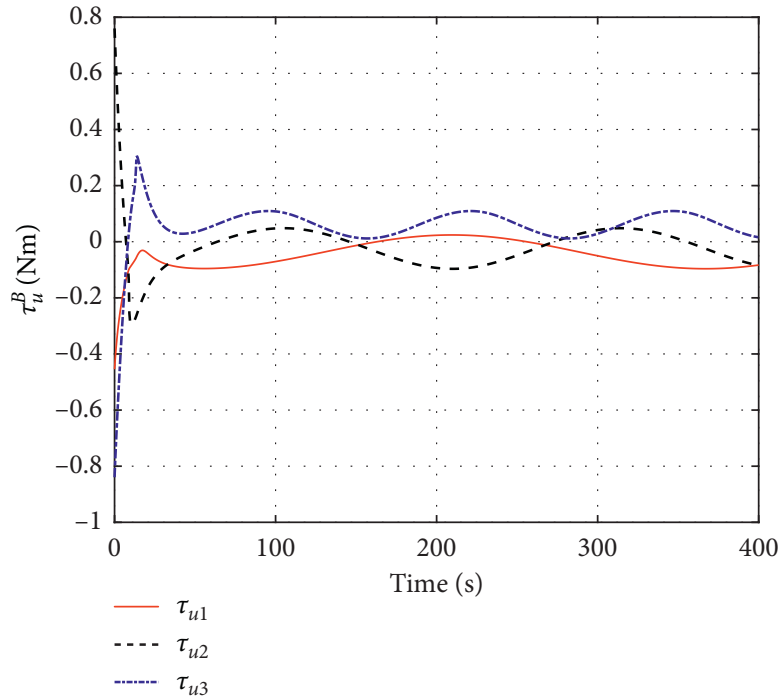

(b)

Figure 14: Control torque. (a) Proposed I\&I adaptive controller. (b) SMC.

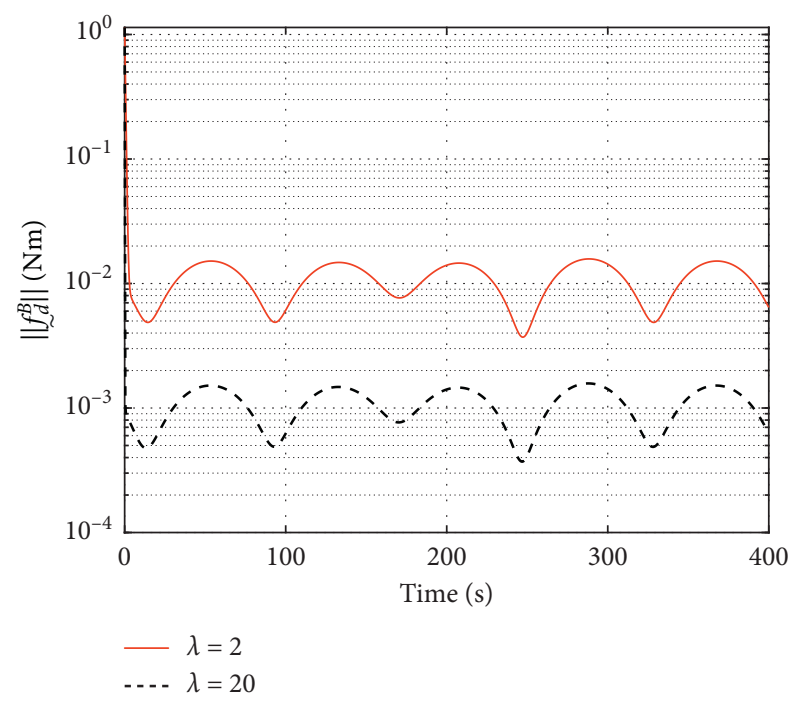

(a)

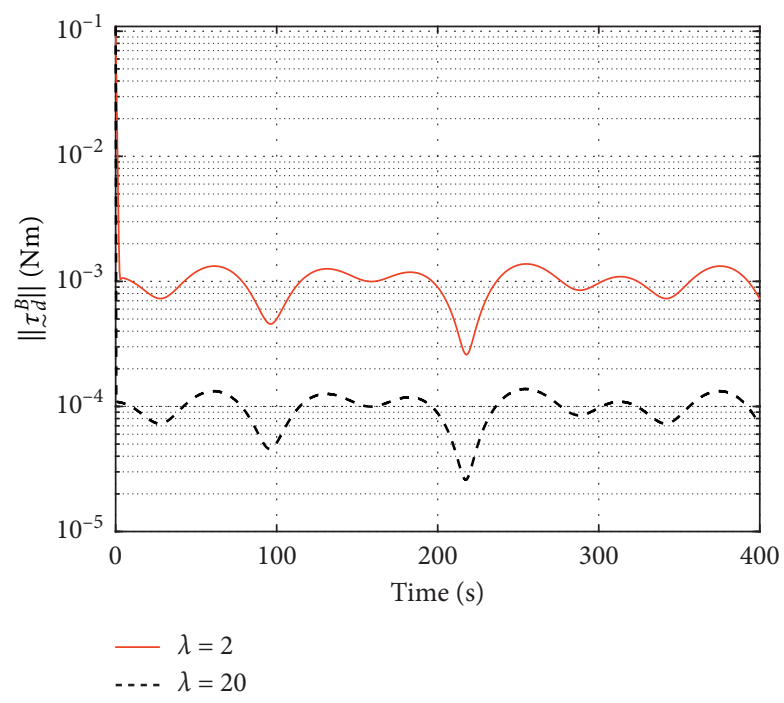

(b)

Figure 15: Disturbance estimation error norms with different $\lambda$.

\section{Conclusion}

The six-degree-of-freedom relative motion control between two rigid spacecraft in the presence of general unknown bounded disturbances is investigated based on dual quaternion description. The newly introduced dual direction cosine matrix enables the integrated relative dynamics to be written in a six-dimensional vector/matrix form. Adopting an adaptive perspective, a noncertainty equivalent disturbance estimator is designed based on the immersion and invariance (I\&I) adaptive control theory. For constant disturbances and time-varying disturbances with bounded first derivatives, the estimation errors exponentially converge to the origin and adjustable small neighbourhood around the origin at the prescribed rate, respectively. Since the I\&Ibased adaption law is driven by the disturbance estimation errors, convergence of the estimation is independent of the applied control law and can realize "parameter lock" when the true values are reached. Combining the disturbance estimation with a proportional-derivative-like pose tracking control law yields the complete I\&I adaptive pose tracking controller. Simulation results demonstrate the effectiveness of the proposed adaptive control scheme in tracking the target pose with high precision under general unknown bounded disturbances. Comparisons with the existing certainty equivalence-based adaptive controller and sliding mode controller illustrate the novel features of the proposed controller and show that owing to the quick and accurate 
estimation of the disturbances, the proposed controller can generate smoother transient performance and higher control precision with less energy cost.

\section{Data Availability}

The data used to support the findings of this study are available from the corresponding author upon request.

\section{Conflicts of Interest}

The authors declare that there are no conflicts of interest regarding the publication of this paper.

\section{Acknowledgments}

This study was supported by the Major Program of the National Natural Science Foundation of China (grant no. 61690210).

\section{References}

[1] K. Xia and W. Huo, "Robust adaptive backstepping neural networks control for spacecraft rendezvous and docking with input saturation," ISA Transactions, vol. 62, no. 3, pp. 249-257, 2016.

[2] J. Shan, "Six-degree-of-freedom synchronised adaptive learning control for spacecraft formation flying," IET Control Theory and Applications, vol. 2, no. 10, pp. 930-949, 2016.

[3] T. Chen, H. Wen, H. Hu, and D. Jin, "Output consensus and collision avoidance of a team of flexible spacecraft for on-orbit autonomous assembly," Acta Astronautica, vol. 121, pp. 271281, 2016.

[4] H. Hakima and M. R. Emami, "Concurrent attitude and orbit control for deorbiter CubeSat," Aerospace Ence and Technology, vol. 97, Article ID 105616, 12 pages, 2020.

[5] D. Lee, A. Sanyal, E. Butcher, and D. Scheeres, "Finite-time control for spacecraft body-fixed hovering over an asteroid," IEEE Transactions on Aerospace and Electronic Systems, vol. 51, no. 1, pp. 506-520, 2015.

[6] J. Sun and Z. Geng, "Formation tracking control for multiple rigid bodies on matrix Lie groups: a system decomposition approach," International Journal of Robust and Nonlinear Control, vol. 27, no. 18, pp. 4188-4207, 2017.

[7] C. K. Verginis, A. Nikou, and D. V. Dimarogonas, "Robust formation control in $\mathrm{SE}(3)$ for tree-graph structures with prescribed transient and steady state performance," Automatica, vol. 103, pp. 538-548, 2018.

[8] K. Subbarao and S. Welsh, "Nonlinear control of motion synchronization for satellite proximity operations," Journal of Guidance, Control, and Dynamics, vol. 31, no. 5, pp. 12841294, 2008.

[9] R. Kristiansen, P. J. Nicklasson, and J. T. Gravdahl, "Spacecraft coordination control in 6DOF: integrator backstepping vs passivity-based control," Automatica, vol. 44, no. 11, pp. 2896-2901, 2008.

[10] L. Sun, W. Huo, and Z. Jiao, "Adaptive nonlinear robust relative pose control of spacecraft autonomous rendezvous and proximity operations," ISA Transactions, vol. 67, pp. 47-55, 2017.

[11] M. Xin and H. Pan, "Nonlinear optimal control of spacecraft approaching a tumbling target," Aerospace Science and Technology, vol. 15, no. 2, pp. 79-89, 2019.
[12] X. Lin, X. Shi, S. Li, S. K. Nguang, and L. Zhang, "Nonsingular fast terminal adaptive neuro-sliding mode control for spacecraft formation flying systems," Complexity, vol. 2020, Article ID 5875191, 15 pages, 2020.

[13] J. Funda and R. P. Paul, "A computational analysis of screw transformations in robotics," IEEE Transactions on Robotics and Automation, vol. 6, no. 3, pp. 348-356, 1990.

[14] N. A. Aspragathos and J. K. Dimitros, "A comparative study of three methods for robot kinematics," IEEE Transactions on Systems, Man and Cybernetics, Part B (Cybernetics), vol. 28, no. 2, pp. 135-145, 1998.

[15] X. Wang and H. Zhu, "On the comparisons of unit dual quaternion and homogeneous transformation matrix," Advances in Applied Clifford Algebras, vol. 24, no. 1, pp. 213-229, 2014.

[16] F. Thomas, "Approaching dual quaternions from matrix algebra," IEEE Transactions on Robotics, vol. 30, no. 5, pp. 1037-1048, 2014.

[17] M. Schilling, "Universally manipulable body models-dual quaternion representations in layered and dynamic MMCs," Autonomous Robots, vol. 30, no. 4, pp. 399-425, 2011.

[18] Z. Fu, J. Pan, E. Spyrakos-Papastavridis, X. Chen, and M. Li, "A dual quaternion-based approach for coordinate calibration of dual robots in collaborative motion," IEEE Robotics and Automation Letters, vol. 5, no. 3, pp. 4086-4093, 2020.

[19] Y. Wu, X. Hu, D. Hu, T. Li, and J. Lian, "Strapdown inertial navigation system algorithms based on dual quaternions," IEEE Transactions on Aerospace and Electronic Systems, vol. 41, no. 1, pp. 110-132, 2005.

[20] B. Qiao, S. Tang, K. Ma, and Z. Liu, "Relative position and attitude estimation of spacecrafts based on dual quaternion for rendezvous and docking," Acta Astronautica, vol. 91, pp. 237-244, 2013.

[21] N. Filipe, M. Kontitsis, and P. Tsiotras, "Extended kalman filter for spacecraft pose estimation using dual quaternions," Journal of Guidance Control and Dynamics, vol. 38, no. 9, pp. 1641-1625, 2015.

[22] N. T. Dantam, "Robust and efficient forward, differential, and inverse kinematics using dual quaternions," International Journal of Robotics Research, 19 pages, 2020.

[23] V. Brodsky and M. Shoham, "Dual numbers representation of rigid body dynamics," Mechanism and Machine Theory, vol. 35, no. 5, pp. 693-718, 2000.

[24] J.-Y. Wang, H.-Z. Liang, Z.-W. Sun, S.-N. Wu, and S.-J. Zhang, "Relative motion coupled control based on dual quaternion," Aerospace Science and Technology, vol. 25, no. 1, pp. 102-113, 2013.

[25] N. Filipe and P. Tsiotras, "Simultaneous position and attitude control without linear and angular velocity feedback using dual quaternions," in Proceedings of the American Control Conference, pp. 4808-4813, Washington, DC, USA, June 2013.

[26] X. Wang and C. Yu, "Unit dual quaternion-based feedback linearization tracking problem for attitude and position dynamics," Systems \& Control Letters, vol. 62, no. 3, pp. 225-233, 2013.

[27] N. Filipe and P. Tsiotras, "Adaptive position and attitudetracking controller for satellite proximity operations using dual quaternions," Journal of Guidance, Control, and Dynamics, vol. 38, no. 4, pp. 566-577, 2015.

[28] H. Gui and G. Vukovich, "Dual-quaternion-based adaptive motion tracking of spacecraft with reduced control effort," Nonlinear Dynamics, vol. 83, no. 1-2, pp. 597-614, 2016. 
[29] J. Wang and Z. Sun, "6-DOF robust adaptive terminal sliding mode control for spacecraft formation flying," Acta Astronautica, vol. 73, pp. 76-87, 2012.

[30] J. Wang, H. Liang, Z. Sun, S. Zhang, and M. Liu, "Finite-time control for spacecraft formation with dual-number-based description," Journal of Guidance, Control, and Dynamics, vol. 35, no. 3, pp. 950-962, 2012.

[31] H. Dong, Q. Hu, M. I. Friswell, and G. Ma, "Dual-quaternionbased fault-tolerant control for spacecraft tracking with finitetime convergence," IEEE Transactions on Control Systems Technology, vol. 25, no. 4, pp. 1231-1242, 2016.

[32] J. Yang and E. Stoll, "Adaptive sliding mode control for spacecraft proximity operations based on dual quaternions," Journal of Guidance, Control, and Dynamics, vol. 42, no. 11, pp. 2356-2368, 2019.

[33] A. Sanyal, A. Fosbury, N. Chaturvedi, and D. S. Bernstein, "Inertia-free spacecraft attitude tracking with disturbance rejection and almost global stabilization," Journal of Guidance, Control, and Dynamics, vol. 32, no. 4, pp. 1167-1178, 2009.

[34] Q. Dang, H. Gui, M. Xu, and H. Wen, "Dual-quaternion immersion and invariance velocity observer for controlling asteroid-hovering spacecraft," Acta Astronautica, vol. 161, pp. 304-312, 2019.

[35] H. Dong, Q. Hu, M. R. Akella, and F. Mazenc, "Partial lyapunov strictification: dual-quaternion-based observer for 6DOF tracking control," IEEE Transactions on Control Systems Technology, vol. 27, no. 6, pp. 2453-2469, 2019.

[36] D. Seo and M. R. Akella, "High-performance spacecraft adaptive attitude-tracking control through attracting-manifold design," Journal of Guidance, Control, and Dynamics, vol. 31, no. 4, pp. 884-891, 2008.

[37] K. W. Lee and S. N. Singh, "Multi-input noncertaintyequivalent adaptive control of an aeroelastic system," Journal of Guidance, Control, and Dynamics, vol. 33, no. 5, pp. 1451-1460, 2010.

[38] A. Astolfi and R. Ortega, "Immersion and invariance: a new tool for stabilization and adaptive control of nonlinear systems," IEEE Transactions on Automatic Control, vol. 48, no. 4, pp. 590-606, 2003.

[39] R. Ortega, L. Hsu, and A. Astolfi, "Immersion and invariance adaptive control of linear multivariable systems," Systems \& Control Letters, vol. 49, no. 1, pp. 37-47, 2003.

[40] R. Ortega, X. Liu, H. Su, and J. Chu, "Immersion and invariance adaptive control of nonlinearly parameterized nonlinear systems*," IFAC Proceedings Volumes, vol. 43, no. 14, pp. 641-646, 2010.

[41] A. Astolfi, D. Karagiannis, and R. Ortega, Nonlinear and Adaptive Control with Applications, Springer-Verlag, London, UK, 2008.

[42] H. Wen, X. Yue, P. Li, and J. Yuan, "Fast spacecraft adaptive attitude tracking control through immersion and invariance design," Acta Astronautica, vol. 139, pp. 77-84, 2017.

[43] K. W. Lee and S. N. Singh, "Quaternion-based adaptive attitude control of asteroid-orbiting spacecraft via immersion and invariance," Acta Astronautica, vol. 167, pp. 164-180, 2020.

[44] D. Xia, X. Yue, H. Wen, and L. Li, "Immersion and invariance adaptive tracking control for robot manipulators with a novel modified scaling factor design," International Journal of Adaptive Control and Signal Processing, vol. 34, no. 1, pp. 110-125, 2019.

[45] T. Xu, J. Xu, and X. Zhang, "Inertia-free computation efficient immersion and invariance adaptive tracking control for
Euler-Lagrange mechanical systems with parametric uncertainties," Advances in Space Research, vol. 66, no. 8, pp. 1902-1910, 2020.

[46] S. Dongeun, "Fast adaptive pose tracking control for satellites via dual quaternion upon non-certainty equivalence principle," Acta Astronautica, vol. 115, pp. 32-39, 2015.

[47] J. Hu and H. Zhang, "Immersion and invariance based command-filtered adaptive backstepping control of VTOL vehicles," Automatica, vol. 49, no. 7, pp. 2160-2167, 2013.

[48] K. W. Lee and S. N. Singh, "Noncertainty-equivalence spacecraft adaptive formation control with filtered signals," Journal of Aerospace Engineering, vol. 30, no. 5, 12 pages, Article ID 04017029, 2017.

[49] K. W. Lee and S. N. Singh, "Immersion-and invariance-based adaptive control of asteroid-orbiting and -hovering spacecraft," Journal of the Astronautical Sciences, vol. 66, no. 3, pp. 5237-5553, 2019. 